\title{
Superluminality, black holes and EFT
}

\author{
Garrett Goon $^{a}$ and Kurt Hinterbichler ${ }^{b}$ \\ ${ }^{a}$ Department of Applied Mathematics and Theoretical Physics, \\ Cambridge University, Cambridge, CB3 0WA, U.K. \\ ${ }^{b}$ CERCA, Department of Physics, Case Western Reserve University, \\ 10900 Euclid Ave, Cleveland, OH 44106, U.S.A. \\ E-mail: gg399@cam.ac.uk, kurt.hinterbichler@case.edu
}

ABSTRACT: Under the assumption that a UV theory does not display superluminal behavior, we ask what constraints on superluminality are satisfied in the effective field theory (EFT). We study two examples of effective theories: quantum electrodynamics (QED) coupled to gravity after the electron is integrated out, and the flat-space galileon. The first is realized in nature, the second is more speculative, but they both exhibit apparent superluminality around non-trivial backgrounds. In the QED case, we attempt, and fail, to find backgrounds for which the superluminal signal advance can be made larger than the putative resolving power of the EFT. In contrast, in the galileon case it is easy to find such backgrounds, indicating that if the UV completion of the galileon is (sub)luminal, quantum corrections must become important at distance scales of order the Vainshtein radius of the background configuration, much larger than the naive EFT strong coupling distance scale. Such corrections would be reminiscent of the non-perturbative Schwarzschild scale quantum effects that are expected to resolve the black hole information problem. Finally, a byproduct of our analysis is a calculation of how perturbative quantum effects alter charged Reissner-Nordstrom black holes.

KeYwords: Black Holes, Effective field theories, Classical Theories of Gravity

ARXIV EPRINT: 1609.00723 


\section{Contents}

1 Introduction and summary 2

2 The QED effective theory $\quad 8$

3 The Drummond-Hathrell problem $\quad 10$

$\begin{array}{lll}3.1 & \text { Black hole setup } & 10\end{array}$

$\begin{array}{lll}3.2 & \text { Geometric optics analysis } & 11\end{array}$

4 Building up QED superluminality 14

4.1 Simple attempts: large $N_{f}$, small $r_{s}$ and photon orbits $\quad 15$

$\begin{array}{ll}4.2 \text { A ladder of black holes: large } N_{\mathrm{BH}} & 16\end{array}$

5 Black hole ladder analysis $\quad 16$

$\begin{array}{lll}5.1 & \text { Einstein-Maxwell background } & 17\end{array}$

$\begin{array}{ll}5.2 \text { Quantum corrections } & 17\end{array}$

5.2.1 Feynman diagram estimation: RN black holes 18

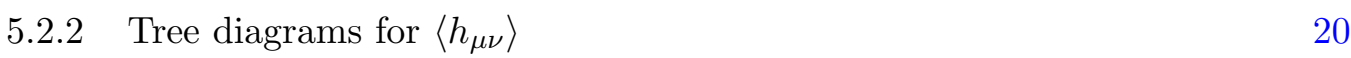

5.2.3 $\left\langle h_{\mu \nu}\right\rangle$ from photon and graviton loops 22

5.2.4 Subtleties of gauge loops 23

5.2.5 Combining all effects 24

5.3 Tunnel dynamics and distance advance 26

$\begin{array}{lll}\text { 5.3.1 Tunnel dynamics } & 26\end{array}$

$\begin{array}{lll}5.3 .2 & \text { Distance advance } & 28\end{array}$

$\begin{array}{lll}\text { 5.3.3 Variations } & 29\end{array}$

$\begin{array}{lll}\text { 5.3.4 Weak gravity conjecture } & 31\end{array}$

$\begin{array}{lll}\text { 5.3.5 Polarization rotation } & 31\end{array}$

6 Galileon superluminality $\quad 32$

6.1 Background solution 33

6.2 Geometric optics analysis 33

6.3 Racing $\delta \pi$ against a photon 33

$\begin{array}{lll}7 & \text { Conclusions } & 34\end{array}$

A Velocity dependent forces $\quad 35$

B Effects of $F^{2 n} / m_{e}^{4 n-4}$ operators on $c_{s} \quad 37$

$\begin{array}{lll}\text { C One-loop potential from massless fields } & 37\end{array}$ 


\section{Introduction and summary}

It is expected that a reasonable physical theory should pass a few baseline "consistency" tests. One often invoked criteria is freedom from superluminalities; no signal should travel with a velocity exceeding the speed of light. Nevertheless, there exist interesting effective field theories (EFTs), including some which we know to be realized in nature, which display apparent superluminal behavior. No true consistency condition should rule out a theory realized in nature, so if freedom from superluminalities is indeed such a condition, the effect must be spurious, i.e. outside the regime of validity of the theory. The goal of this paper is to gain a better understanding of when, or whether, superluminality can be acceptable in the context of an EFT.

Though often touted as a failure of "consistency," or as "acausality," one should keep in mind that superluminality does not necessarily imply closed time-like curves (time machines) [1-4], and even closed time-like curves do not necessarily imply inconsistency [5]. Nevertheless, we may still proceed under the conservative assumption that a fundamental UV theory should not allow superluminal signaling, an assumption nature has not yet shown us a violation of, and ask what this implies for the effective theory. In this paper, this topic is studied in the context of two specific EFTs, one realized in nature and the other speculative.

Our example realized in nature will be quantum electrodynamics (QED) coupled to gravity. The $\mathrm{UV}^{1}$ action is a minimally coupled Dirac fermion ${ }^{2}$

$$
S_{\mathrm{QED}}=\int \mathrm{d}^{4} x \sqrt{-g}\left[\frac{M_{p}^{2}}{2} R-\frac{1}{4 e^{2}} F_{\mu \nu}^{2}+\bar{\psi}\left(i \not D-m_{e}\right) \psi\right],
$$

where $m_{e}$ is the mass of the fermion. Integrating out the fermion generates an EFT for a photon which is non-minimally coupled to gravity:

$$
S=\int \mathrm{d}^{4} x \sqrt{-g}\left[\frac{M_{p}^{2}}{2} R-\frac{1}{4 e^{2}} F_{\mu \nu}^{2}+\frac{1}{4 \pi^{2}} \frac{1}{360 m_{e}^{2}} R_{\mu \nu \rho \sigma} F^{\mu \nu} F^{\rho \sigma}+\ldots\right] .
$$

The higher derivative operators are suppressed by the electron mass $m_{e}$, corresponding to the strong coupling distance scale $\sim m_{e}^{-1}$.

These derivative couplings can alter photon (and graviton) propagation on non-trivial backgrounds. In a seminal paper, Drummond and Hathrell [6] demonstrated that in the EFT (1.2) photons can propagate on black hole (BH) backgrounds with a speed ${ }^{3} c_{s}>1$. The setup is shown in figure 1. Consider a photon traveling in the angular direction at an

\footnotetext{
${ }^{1}$ UV here means valid up to the Planck scale, not truly UV, but we know this must be UV completed in some way, since it's realized in nature.

${ }^{2}$ Of course, precision tests confirm to high accuracy the $\left\{A_{\mu}, \psi\right\}$ sector of the theory and the classic tests of GR confirm the Einstein-Hilbert term, but little is known about possible non-minimal couplings between $\left\{A_{\mu}, \psi\right\}$ and $g_{\mu \nu}$ and other higher order interactions. We assume these are negligible in the UV action.

${ }^{3}$ Used in the context of photon propagation, "superluminal" is perhaps not the best word. "Superluminal" here means the photon travels faster than some hypothetical massless test particle which is coupled minimally to the theory, or equivalently, that the photon travels outside the light-cone of the background metric.
} 


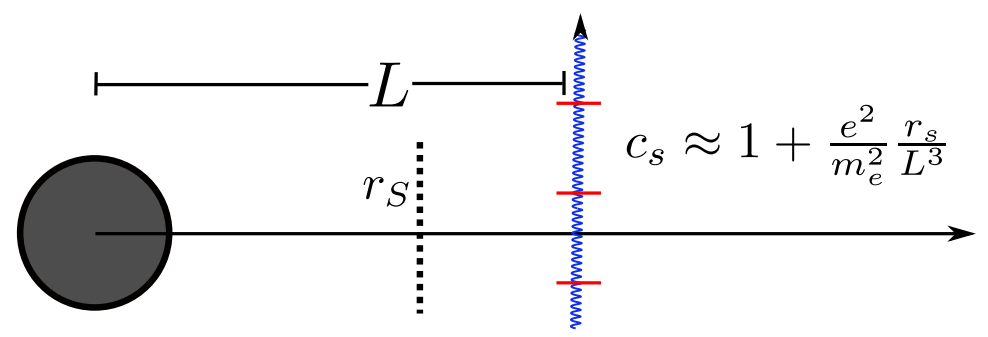

Figure 1. Sketch of the Drummond-Hathrell problem [6]. A photon passes a distance $L$ from a Schwarzschild $\mathrm{BH}$ of radius $r_{s}$. If the polarization is pointing radially outwards, as indicated by the red lines, the EFT (1.2) gives a superluminal speed.

impact parameter $L$ from a Schwarzschild BH with Schwarzschild radius $r_{s}$. The photon's polarization is pointing radially. From the EFT (1.2), the photon's speed $c_{s}=1+\delta c_{s}$ can be estimated to be of order

$$
\delta c_{s} \sim \frac{\bar{R}_{\mu \nu \rho \sigma}}{m_{e}^{2}} \approx \frac{e^{2}}{m_{e}^{2}} \frac{r_{s}}{L^{3}}
$$

Only for these kinematics do we get $c_{s}>1$. When the polarization vector points azimuthally the speed is subluminal $c_{s}<1$ and radially propagating photons have $c_{s}=1$ regardless of polarization.

This effect is a bit of a longstanding oddity. The expectation is that full QED (1.1) should not allow for superluminal propagation [7], so why is it displayed in the effective theory? There have been many studies of the problem from an array of angles, coming to a variety of conclusions (see e.g. $[8-14]^{4}$ ).

One possible resolution was already pointed out in the original paper [6]: this unexpected effect is tiny. Specifically, as the photon traverses its entire path across the black hole, the effect generates a cumulative distance advance of order ${ }^{5}$

$$
\Delta d \approx \delta c_{s} \times L \approx m_{e}^{-1}\left(\frac{e^{2}}{m_{e}} \frac{r_{s}}{L^{2}}\right) \ll m_{e}^{-1},
$$

i.e. a length parametrically smaller than the cutoff distance of the theory (the inverse electron mass $m_{e}^{-1}$ ) for any valid choices of $e, r_{s}$ and $L$. Because this small distance advance is below the resolving power of the EFT, the superluminality cannot be said to be a "real" effect, at least in this particular setup. This is an indication that the apparent superluminality is simply an artifact of the approximations made when using the effective theory.

\footnotetext{
${ }^{4}$ Prominent in the literature is the work of Shore, a former student of Drummond, who, with Daniels, extended the calculation to Reissner-Nordstrom [15] and Kerr [16] and, with Hollowood and collaborators, studied the nature of QED photon trajectories with an emphasis on carefully studying the fate of the effect in the full UV theory [17-26].

${ }^{5}$ For example, taking a Standard Model electron and solar mass black hole, the distance advance is at least as small as $\Delta d \lesssim 10^{-31} \mathrm{~m}$, much smaller than the cutoff $m_{e}^{-1} \sim 10^{-13} \mathrm{~m}$ and not so far from the Planck length $l_{p} \sim 10^{-34} \mathrm{~m}$.
} 
If the superluminality is an artifact of the EFT expansion, it must be cured in the full theory. ${ }^{6}$ One way this can happen is as follows: the velocities we are implicitly talking about in the effective field theory are group velocities, $v_{g}=\frac{\mathrm{d} \omega}{\mathrm{d} k}$ (which happen to be same as the phase velocities $v_{p}=\frac{\omega}{k}$ for the massless theories we are talking about since the dispersion relations are, to lowest order, linear $\omega \propto k$ ). However, the speed at which actual information carrying signals can be sent is instead given by the front velocity which tracks the movement of the sharp boundary between regions of zero and non-zero signal [3032]. A perfect description of this discontinuous surface is inaccessible to the perturbative EFT, ${ }^{7}$ since it cannot resolve spatial distances smaller than its strong coupling distance scale. If the accumulated distance advance along any particle path on any background calculated using any EFT notion of velocity is smaller than the resolution of the EFT, we may attribute any discrepancy between the EFT velocity and some expected front velocity in the full theory to the inherent fuzziness of the EFT, and there is no cause for concern.

Thus the question is the following: does $\Delta d \ll m_{e}^{-1}$ persist for all possible backgrounds and setups? Clearly, there are two possibilities:

1. There exists no setup describable within the QED EFT which generates a macroscopic distance advance, $\Delta d \gg m_{e}^{-1}$.

2. If we work hard enough, we can construct a setup in the QED EFT which generates a macroscopic distance advance, $\Delta d \gg m_{e}^{-1}$.

If the first scenario were true, then our naive expectations about the EFT would be met: the full UV theory can be free of superluminalities and the effective description can be used and trusted all the way to distances $\sim m_{e}^{-1}$ without worrying about the spurious superluminality. If the second scenario were true, then we would have a background with some scale $\Lambda^{-1} \gg m_{e}^{-1}$ over which we would have superluminality. In this case, under the assumption that the full UV theory is (sub)luminal, strong quantum effects or extra degrees of freedom must come in at the background-dependent scale $\Lambda$, sooner than the naive cutoff $m_{e}$, in order to cure the superluminality.

In either case, when studying an EFT with an unknown UV completion, the low-energy superluminality never acts as a "consistency test" to rule out the effective theory. Instead, it simply tells us when strong coupling or UV degrees of freedom must enter if the full theory is to be (sub)luminal.

We expect that the first scenario must be true for the QED effective theory. Since the UV theory is known, we know that quantum effects and extra degrees of freedom should not become important until the distance $\sim m_{e}^{-1}$. Thus it should be impossible to find a background or setup with $\Delta d \gg m_{e}^{-1}$.

In what follows, we find strong evidence for the first scenario: it is extremely difficult to generate $\Delta d>m_{e}^{-1}$ in QED. Though we will not be able to analyze every possible scenario, and are therefore unable to elevate our results to the level of a theorem, we will

\footnotetext{
${ }^{6}$ EFTs also protect themselves against other apparent pathologies, such as ghosts arising from higher derivative operators in the EFT [27-29].

${ }^{7}$ Dispersion relations relate the front velocity to IR quantities, but their use in curved space is subtle [18].
} 
build setups which go to great lengths to try to magnify the superluminal effect, yet still fall short of accomplishing $\Delta d>m_{e}^{-1}$. Specifically, we attempt to build up the distance advance by passing the photon through an enormous number of black hole pairs. The black holes are taken to be be nearly extremal Reissner-Nordstrom (RN), so that the only forces which destabilize the pairs of BHs are those generated from loops.

The construction provides a rich demonstration of the conspiracies which must occur in order to prevent the generation of macroscopic distance advances. There are many competing scales to balance and effects to account for, and only when they are all included do we find that macroscopic superluminality in QED is avoided. The thought experiments give an idea of how extreme and contrived any scenario generating $\Delta d>m_{e}^{-1}$ would likely need to be.

Our other example of a superluminal EFT, the more speculative one, is that of the galileon, a single scalar $\pi(x)$ whose defining property is a global shift symmetry $\pi(x) \rightarrow \pi(x)+b+c_{\mu} x^{\mu}$ with constant $b, c_{\mu}$ [33]. Galileons have been widely studied as a particularly interesting class of EFTs. For example, they capture much of the interesting phenomenology of IR modified gravity theories including the Dvali-GabadadzePorrati (DGP) $[34,35]$ braneworld model, the de Rham-Gabadadze-Tolley (dRGT) theory of massive General Relativity (GR) [36] (see [32, 37, 38] for reviews) and other brane-world setups [39-42]. They also possess many interesting properties in their own right, such as Vainshtein screening [43, 44] and strong non-renormalization theorems [35, 45].

The galileons come in many different forms and generalizations (e.g. [39-42, 46-48]), but the simplest example is the cubic galileon

$$
\mathcal{L}=-\frac{1}{2}(\partial \pi)^{2}-\frac{1}{\Lambda^{3}}(\square \pi)(\partial \pi)^{2}+\frac{1}{M_{p}} \pi T_{\mu}^{\mu},
$$

where $\Lambda$ is the strong coupling scale of the EFT. We have coupled it with gravitational strength to a matter source ${ }^{8}$ which is the trace of the matter stress tensor. This is the coupling that occurs in most IR modified gravity applications of the galileon.

In the presence of a static point mass $T_{\mu}^{\mu}(x) \sim M \delta^{3}(\vec{x})$, a non-trivial spherically symmetric field profile $\bar{\pi}(r)$ develops. This creates a potential, $V \sim \frac{1}{M_{p}} \bar{\pi}(r)$, felt by matter. Far from the source, the quadratic kinetic term of (1.5) dominates over the cubic term and we have $\bar{\pi}(r) \sim \frac{M}{M_{p}} \frac{1}{r}$, resulting in a gravitational strength fifth force $V \sim \frac{M}{M_{p}^{2}} \frac{1}{r}$.

If this force persisted at all distance scales, the model would be ruled out phenomenologically. However, the galileon has a highly efficient screening mechanism, known as the Vainshtein mechanism [43] (see [44] for a review), active in regions sufficiently close to the source. There is a distance scale $r_{V} \equiv \Lambda^{-1}\left(M / M_{p}\right)^{1 / 3}$ the "Vainshtein radius" of the source, where the cubic interaction in (1.5) becomes as important as the quadratic kinetic term and the field profile changes significantly. At distances much smaller than the Vainshtein radius, the cubic term dominates and we have $\bar{\pi}(r) \sim\left(\frac{r}{r_{V}}\right)^{3 / 2} \frac{M}{M_{p}} \frac{1}{r}$, greatly suppressing the potential $V \sim \frac{M}{M_{p}^{2}} \frac{1}{r}\left(\frac{r}{r_{V}}\right)^{3 / 2}$, see figure 2. This effect is crucial for the compatibility of galileon, DGP and dRGT theories with solar system test of gravity. ${ }^{9}$

\footnotetext{
${ }^{8}$ The matter coupling might not appear to be invariant under the galileon symmetry, but it is in the
} 


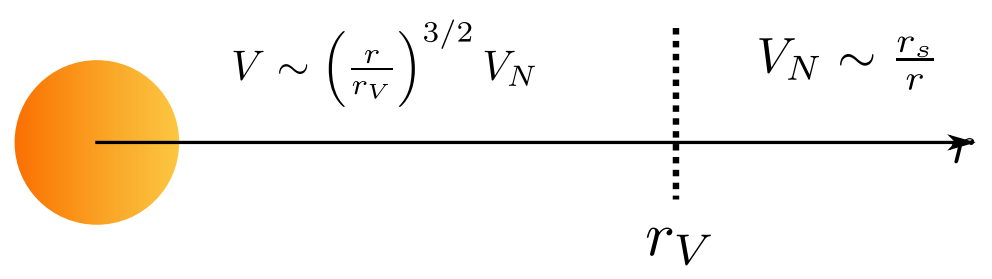

Figure 2. Sketch of the Vainshtein mechanism for the cubic galileon (1.5) around the Sun. Far from a source, the cubic galileon generates a potential of Newtonian strength $V \sim V_{N} \sim r_{s} / r$. Below the non-linear distance scale $r_{V} \sim \Lambda^{-1}\left(M / M_{p}\right)^{1 / 3}$ screening becomes effective and the fifth force is suppressed by a factor of $\left(r / r_{V}\right)^{3 / 2}$.

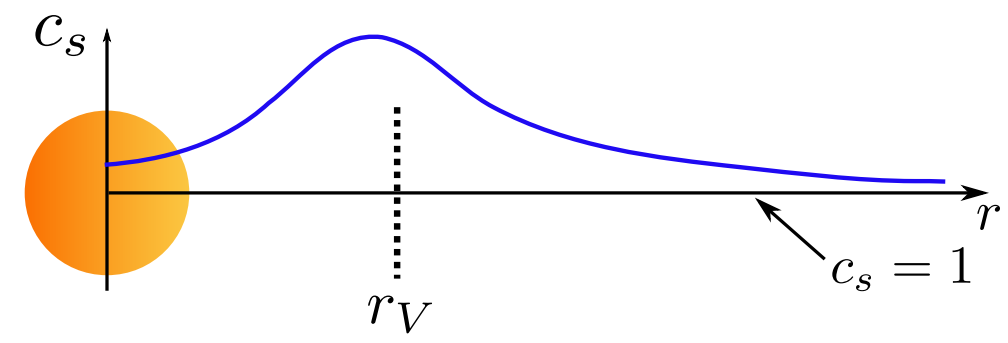

Figure 3. Sketch of superluminality induced by the Sun for the cubic galileon. Radially moving perturbations travel with a position dependent speed of sound, indicated by the blue curve. The horizontal axis represents $c_{s}=1$. For the purely cubic galileon, $c_{s} \geq 1$ when $r \lesssim r_{V}$, though $c_{s} \rightarrow 1$ at large $r$.

The same non-linearities responsible for screening also generate superluminal sound speeds for perturbations about the $\bar{\pi}(r)$ background [33, 50]. This effect is quite generic to generalizations of the galileons [51-56] and seems to be a generic feature of theories possessing Vainshtein screening (there are exceptions, however [57, 58]). Specifically, radially propagating perturbations around the background $\bar{\pi}(x)$ acquire a speed $c_{s}>1$ at distances $r \gtrsim r_{V}$. Expanding the cubic interaction (1.5) about the background allows us to read off the approximate expression for the sound speed $c_{s}=1+\delta c_{s}$,

$$
\delta c_{s} \sim \frac{\partial^{2} \bar{\pi}(r)}{\Lambda^{3}} \approx\left(\frac{r_{V}}{r}\right)^{3}
$$

The sign of $\delta c_{s}$ turns out to be positive and (1.6) represents an $\mathcal{O}(1)$ effect near $r_{V}$, with $c_{s}$ settling back to unity as $r \rightarrow \infty$, see figure 3. (By including higher order galileon operators it is possible to turn $c_{s}$ subluminal at distances close to the source so that significant superluminality only exists at $r \sim r_{V}$ [33].) All other perturbations, i.e. those in the angular directions, propagate subluminally.

\footnotetext{
limit that the matter is non-dynamical.

${ }^{9}$ Consider galileons in the Solar System. In models where the size of the IR modification is chosen to account for the present accelerated expansion, one typically has $\Lambda^{-1} \sim \mathcal{O}\left(10^{3} \mathrm{~km}\right) \sim \mathcal{O}\left(10^{-11} \mathrm{pc}\right)$, meaning that the Sun's Vainshtein radius is $r_{V}^{\odot} \sim \mathcal{O}(200 \mathrm{pc})$. Since the Solar System's radius is $\sim \mathcal{O}\left(10^{-4} \mathrm{pc}\right)$, any local galileon potential is suppressed by a factor of at least $\sim 10^{-9}$ relative to to the usual Newtonian result, making all effects minuscule, but still possibly detectible with precise enough measurements [49].
} 
This superluminality has caused much worry, and is thought to imply similar superluminalities within the full DGP [59] and dRGT [60] theories. ${ }^{10}$ In contrast to QED, the galileon superluminality generates macroscopic distance advances, as compared to the galileon strong coupling distance scale $\Lambda^{-1}$. Sending a galileon signal from near the Vainshtein radius to infinity, the distance advance is of order [61]

$$
\Delta d \approx \int_{\sim r_{V}}^{\infty} \mathrm{d} r \delta c_{s} \approx \int_{\sim r_{V}}^{\infty} \mathrm{d} r\left(\frac{r_{V}}{r}\right)^{3} \approx r_{V} \approx \Lambda^{-1}\left(M / M_{p}\right)^{1 / 3}
$$

which is parametrically larger than the scale $\Lambda^{-1}$ for any large source. ${ }^{11}$ Therefore, while the two scenarios have many superficial similarities, they are qualitatively different in an important way: galileons and QED generate distance advances which are parametrically larger and smaller than the naive cutoffs of the EFTs in the two cases, respectively.

For the galileons, and to the extent that they captures infrared modifications of gravity, this would indicate that if it is possible to fix the galileon superluminality, the cure will be of a qualitatively different type than the prescription for QED. Non-perturbative effects will have to become important at very large distance scales of order the Vainshtein radius, much larger than the naive strong coupling distance scale $\Lambda^{-1}$. The Vainshtein solutions behave in many ways like black holes in general relativity, with the Vainshtein radius playing the role of the Schwarzschild radius. From the black hole information paradox, firewall paradox, etc., there are many reasons to believe that quantum effects near the black hole horizon should become important, causing the local effective field theory expectations to fail, despite the fact that curvatures are much smaller than the naive Planck scale cutoff [62-65]. The same may be true of the galileons, and there are indications that this is the case $[66,67]$, meaning they could potentially serve as a toy model [68] of the firewall paradox [65].

Finally, in a theory with gravity, there are strictly speaking no local observables, and it might be objected that local superluminality of the type we have been implicitly discussing is not a sharp observable from which we can draw sharp conclusions. However, all of the above can be phrased in terms of asymptotic observables, i.e. cumulative time advances measured by sending a signal in from infinity in an asymptotically flat solution and watching for when it comes out at the other side of infinity. We will thus consider only scenarios which can in principle be viewed as this kind of asymptotic scattering experiment, and hence represent sharp observables even in the presence of gravity.

Conventions. Our metric and curvature conventions are those of Carroll [69] (equivalently, Misner, Thorne and Wheeler [70]): we work in mostly plus signature, $\eta_{\mu \nu}=$ $(-,+,+,+)$ and use the curvature conventions

$$
\begin{aligned}
\Gamma_{\mu \nu}^{\lambda} & =\frac{1}{2} g^{\lambda \sigma}\left[\partial_{\mu} g_{\nu \sigma}+\partial_{\nu} g_{\sigma \mu}-\partial_{\sigma} g_{\mu \nu}\right] \\
R_{\sigma \mu \nu}^{\rho} & =\partial_{\mu} \Gamma_{\nu \sigma}^{\rho}-\partial_{\nu} \Gamma_{\mu \sigma}^{\rho}+\Gamma_{\mu \lambda}^{\rho} \Gamma_{\nu \sigma}^{\lambda}-\Gamma_{\nu \lambda}^{\rho} \Gamma_{\mu \sigma}^{\lambda}, \quad R_{\sigma \nu}=R_{\sigma \rho \nu}^{\rho},
\end{aligned}
$$

\footnotetext{
${ }^{10}$ It has not been directly shown within the full massive theories, however, that there is superluminality within the naive regime of validity of the full massive theories. In addition, there are indications that enforcing certain cosmological boundary conditions may eliminate superluminality outright [57, 58].

${ }^{11}$ For the Sun, we would find $\Delta d \sim 200 \mathrm{pc}$ whereas $\Lambda^{-1} \sim 10^{-11} \mathrm{pc}$.
} 
so that $\left[\nabla_{\mu}, \nabla_{\nu}\right] V^{\rho}=R_{\sigma \mu \nu}^{\rho} V^{\sigma}$. We symmetrize and anti-symmetrize indices with weight one, i.e.

$$
T_{(\mu \nu)}=\frac{1}{2}\left[T_{\mu \nu}+T_{\nu \mu}\right], \quad T_{[\mu \nu]}=\frac{1}{2}\left[T_{\mu \nu}-T_{\nu \mu}\right] .
$$

Greek indices run over all of spacetime $\mu \in\{0,1,2,3\}$ and Latin indices run over space $i \in\{1,2,3\}$ (we work in $d=4$ throughout). The Planck mass conventions are $M_{p}^{2} \equiv$ $1 / l_{p}^{2} \equiv\left(8 \pi G_{N}\right)^{-1}$. The Schwarzschild radius for a black hole of mass $M$ is $r_{s}=\frac{M}{4 \pi M_{p}^{2}}$. The distance scale associated to the charge of a charged black hole is defined to be $r_{q}=\frac{Q}{\pi \sqrt{8} M_{p}}$, so that extremal Reissner-Nordstrom black holes satisfy $r_{q}=r_{s}$. The Vainshtein radius for a source of mass $M$ is $r_{V}=\Lambda^{-1}\left(M / M_{p}\right)^{1 / 3}$. Often we will rewrite the electron mass $m_{e}$ in favor of the length scale $r_{e} \equiv m_{e}^{-1}$, which is (roughly) the cutoff of the EFT.

\section{The QED effective theory}

We start with a short review of the QED EFT and discuss its expected regimes of validity. The EFT is constructed by integrating out the electron from the QED action,

$$
\exp i S_{\mathrm{eff}}\left[g_{\mu \nu}, A_{\mu}\right] \equiv \int \mathcal{D} \bar{\psi} \mathcal{D} \psi \exp i S_{\mathrm{QED}}\left[g_{\mu \nu}, A_{\mu}, \bar{\psi}, \psi\right] .
$$

Integrating out the electron is a particularly clean procedure in QED since the UV action is strictly quadratic in fermion fields, so the entire contribution of electrons to the low energy effective action can be written as a single one-loop functional determinant,

$$
S_{\text {eff }}=\int \mathrm{d}^{4} x \sqrt{-g}\left[\frac{M_{p}^{2}}{2} R-\frac{1}{4 e^{2}} F_{\mu \nu}^{2}\right]-i \operatorname{Tr} \ln \left(i \not D-m_{e}\right) .
$$

The effective action is local, expressible as a power series in $\partial$. The precise signs of the various coefficients in the effective action are important for our analysis. Hence, as a check, we re-derived the effective action using two methods: matching amplitudes and directly expanding the functional determinant (using the technique outlined in appendix A of [45]). We find full agreement with the original Drummond-Hathrell result, after accounting for their conventions. ${ }^{12}$

The effective action contains a finite number of divergent terms, while the remaining terms are finite and unambiguous. Up to order $\partial^{4}$, the finite parts of the effective action are

$$
\begin{aligned}
S_{\text {eff }}= & \int \mathrm{d}^{4} x \sqrt{-g}\left(\frac{M_{\mathrm{p}}^{2}}{2} R-\frac{1}{4 e^{2}} F_{\mu \nu}^{2}\right) \\
& +\int \mathrm{d}^{4} x \sqrt{-g} \frac{1}{m_{e}^{2}}\left(a R F_{\mu \nu} F^{\mu \nu}+b R_{\mu \nu} F^{\mu \sigma} F^{\nu}{ }_{\sigma}+c R_{\mu \nu \sigma \tau} F^{\mu \nu} F^{\sigma \tau}+d \nabla_{\mu} F^{\mu \nu} \nabla_{\sigma} F^{\sigma}{ }_{\nu}\right) \\
& \left.+\int \mathrm{d}^{4} x \sqrt{-g} \frac{1}{m_{e}^{4}}\left(y F_{\mu \nu} F_{\sigma \tau} F^{\mu \sigma} F^{\nu \tau}+z\left(F_{\mu \nu} F^{\mu \nu}\right)^{2}\right)\right]+\mathcal{O}\left(\partial^{6}\right),
\end{aligned}
$$

\footnotetext{
${ }^{12}$ In the literature there appears to be some unstated disagreement about the signs in the effective action. For instance, the effective action in [15] has the same signs as the Drummond-Hathrell result [6] and thus claims to be in agreement with their results. However, [15] uses the opposite signature but the same curvature conventions as [6] and therefore should have different signs on the $\sim R F F$ terms in $S_{\text {eff }}$. Other references leave these important conventions unstated entirely. We use the same curvature conventions and opposite metric signature as Drummond-Hathrell.
} 


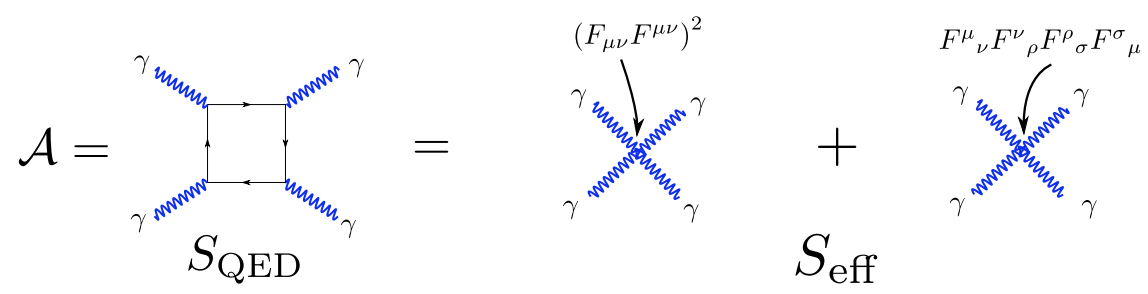

Figure 4. The QED box diagram generates the two $\mathcal{O}\left(F^{4}\right)$ EFT operators in (2.3). Throughout the paper, photons are represented by blue, wavy lines.

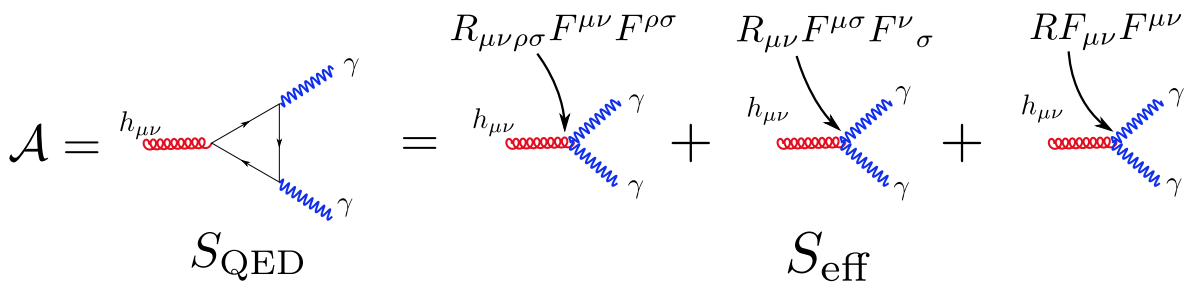

Figure 5. Triangle diagrams generate the three $\sim R F F$ operators in (2.3). Throughout the paper, gravitons will be represented by red, curly lines.

where the $\mathcal{O}(1)$ coefficients are ${ }^{13}$

$$
\left(\begin{array}{l}
a \\
b \\
c \\
d \\
y \\
z
\end{array}\right)=\frac{1}{180} \frac{1}{(4 \pi)^{2}}\left(\begin{array}{c}
5 \\
-26 \\
2 \\
24 \\
14 \\
-5
\end{array}\right) .
$$

The two $\sim F^{4}$ operators arise from the matching shown in figure 4 . The $\sim R F^{2}$ operators arise from the matching in figure 5 .

The divergences appear for the operators ${ }^{14}$

$$
m_{e}^{4} \sqrt{-g}, m_{e}^{2} \sqrt{-g} R, \sqrt{-g} F_{\mu \nu}^{2}, \sqrt{-g} R^{2}, \sqrt{-g} R_{\mu \nu}^{2}
$$

where the coefficients shown reflect the natural scale. In (2.3) we chose counterterms so as to set to zero the coefficient of $\sqrt{-g}$; this is the usual cosmological constant fine tuning. The divergences in $\sqrt{-g} R, \sqrt{-g} F_{\mu \nu}^{2}$ are absorbed into the definitions of $M_{p}$ and $e$, which are now renormalized quantities. The coefficients of the $R^{2}$ operators should also be absorbed into renormalized coefficients. We have not written these operators in the action (2.3) because they play no role in the effects we are interested in as long as its coefficient, $c_{R_{\mu \nu}^{2}}$, obeys $c_{R_{\mu \nu}^{2}} \lesssim\left(\frac{e M_{p}}{m_{e}}\right)^{2}$. The natural size for $c_{R_{\mu \nu}^{2}}$ is $\mathcal{O}(1)$ and we will assume throughout

\footnotetext{
${ }^{13}$ In an abuse of notation, we will refer to every numerical EFT coefficient in (2.4) as being $\mathcal{O}(1)$ throughout the paper, despite the fact that they are numerically $\mathcal{O}\left(10^{-4}\right)$ or $\mathcal{O}\left(10^{-5}\right)$.

${ }^{14}$ The $\sqrt{-g} R_{\mu \nu \rho \sigma}^{2}$ operator also appears, but we can remove it via the Gauss-Bonnet total derivative. It's needed, however, as a counterterm in dimensional regularization.
} 
that $\frac{e M_{p}}{m_{e}}>1$, so no fine tuning is required, given the latter assumption. The condition $\frac{e M_{p}}{m_{e}}>1$ is (one version of) the Weak Gravity Conjecture (WGC) [71]. We will come back later to connections between our work and the WGC.

In principle, the full effective action contains all possible information about low energy fields. For QED, everything we'd ever want to know about processes only involving gravity and light is in $S_{\text {eff }}\left[g_{\mu \nu}, A_{\mu}\right]$. In practice, we necessarily make an approximation by truncating the action: we keep only a few low-dimension operators in $S_{\text {eff }}$ and throw everything else away. It is therefore clear that the truncated EFT cannot be used to study processes at all possible energies. Keeping, for instance, $\left(F_{\mu \nu} F^{\mu \nu}\right)^{2} / m_{e}^{4}$ while neglecting $\left(F_{\mu \nu} F^{\mu \nu}\right)^{3} / m_{e}^{6}$ is only a good approximation to the extent that $F_{\mu \nu} / m_{e}^{2} \ll 1$, with similar criteria holding for the curvature terms. Thus, the range of validity of the truncated QED EFT is restricted to regimes in which energies are smaller than $m_{e}$, distances are larger than $m_{e}^{-1}$ and curvatures and field strengths much smaller than $m_{e}^{2}$. Everything else is below the resolving power of the effective theory. Therefore, given a superluminal $c_{s}>1$ effect in QED which is unable to generate a distance advance larger than $m_{e}^{-1}$, we cannot exclude the possibility that it is a simple artifact of our approximations.

\section{The Drummond-Hathrell problem}

The effective theory (2.3), viewed as a classical theory, admits superluminal propagation around non-trivial backgrounds. This is known as the Drummond-Hathrell problem. In this section we review the original Drummond-Hathrell problem and re-derive the appropriate geometric optics equations for describing the propagation of light in the effective theory.

Note that (2.3) is not a classical theory; it incorporates electron loops but graviton and photon loops have not yet been included. It is not even the one-loop 1PI effective action of the theory (1.1), because there are one-loop diagrams with internal gravitons and photons which have not been included. These diagrams become important in some regimes, and we will discuss their effects later on.

In a theory including gravity defined with flat space asymptotics, it is generally asymptotically defined quantities such as the S-matrix which are the cleanest observables to define. Thus we will ask about superluminality which can in principle be observed asymptotically. We will stick to backgrounds which are asymtotically flat, and ask about asymptotic observables such as the distance advance by which a superluminal photon overtakes a familiar, minimally coupled photon as the two race out to $\infty$ across the asymptotically flat space.

\subsection{Black hole setup}

We will start with a slight variation of the Drummond-Hathrell setup: we use two equal sized black holes, instead of one, so that the photon can pass directly between the pair without curving. ${ }^{15}$ The black holes are separated by a distance much larger than their Schwarzschild radii so that the spacetime is approximately described by the sum of the metric perturbations from each of the black holes. We treat the positions of the black

\footnotetext{
${ }^{15}$ This is the scenario used in appendix A of [72] to discuss Shapiro time delay.
} 

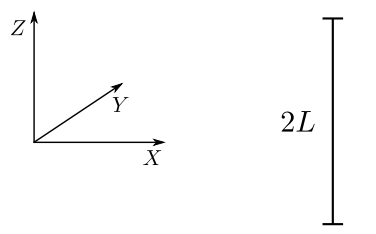

Figure 6. A modified Drummond-Hathrelll setup. The photon passes directly between two black holes a distance $2 L$ apart.

holes as constant. Even though the black holes will attract, the associated time scale is much longer than the time it takes the photon to pass between the pair, so the static approximation is a adequate for our purpose. See figure 6 .

We use isotropic coordinates $x^{\mu}=(t, X, Y, Z)$ and place the two black holes at $\vec{X}_{ \pm}=$ $(0,0, \pm L)$, with $L \gg r_{s}$. Because the black holes are separated by a distance much larger than either of their Schwarzschild radii, the metric in the region between the back holes may be approximated as the sum of the linearized metrics of the two black holes,

$$
\mathrm{d} s^{2}=-\left(1-\frac{r_{s}}{R_{+}}-\frac{r_{s}}{R_{-}}\right) \mathrm{d} t^{2}+\left(1+\frac{r_{s}}{R_{+}}+\frac{r_{s}}{R_{-}}\right) \mathrm{d} \vec{X}^{2}
$$

where $R_{ \pm}^{2}=\left(X^{i}-X_{ \pm}^{i}\right)\left(X^{j}-X_{ \pm}^{j}\right) \delta_{i j}$. Our photon travels in the $X$ direction along the line $Z=Y=0$, and hence its motion is only sensitive to the following non-trivial Riemann curvature components along this path:

\begin{tabular}{|c|c|c|c|c|c|}
\hline$R_{X X t}^{t}$ & $R_{Y Y t}^{t}$ & $R_{Z Z t}^{t}$ & $R_{Y Y X}^{X}$ & $R^{X} Z_{Z X}$ & $R_{Z Z Y}^{Y}$ \\
\hline$\frac{r_{s}\left(L^{2}-2 X^{2}\right)}{\left(L^{2}+X^{2}\right)^{5 / 2}}$ & $\frac{r_{s}}{\left(L^{2}+X^{2}\right)^{3 / 2}}$ & $\frac{r_{s}\left(X^{2}-2 L^{2}\right)}{\left(L^{2}+X^{2}\right)^{5 / 2}}$ & $\frac{r_{s}\left(X^{2}-2 L^{2}\right)}{\left(L^{2}+X^{2}\right)^{5 / 2}}$ & $\frac{r_{s}}{\left(L^{2}+X^{2}\right)^{3 / 2}}$ & $\frac{r_{s}\left(L^{2}-2 X^{2}\right)}{\left(L^{2}+X^{2}\right)^{5 / 2}}$ \\
\hline
\end{tabular}

\subsection{Geometric optics analysis}

Given this background, we may now perform a geometric optics or characteristic analysis to determine the photon trajectories [13, 70,73]. Physically, geometric optics is the regime of wave propagation in which the wave's phase varies much more rapidly than the amplitude, and its characteristic wavelength is much smaller than the typical background curvature scale. Since we are studying photon propagation in the context of the QED EFT, we have the additional restriction that the characteristic wavelength of the wave be much larger than $m_{e}^{-1}$. Since the typical length scale associated to the Riemann curvature is $\mathcal{O}\left(r_{s}\right)$, we are thus working within the wide window between $m_{e}^{-1}$ and $\sim 1 / \sqrt{R_{\mu \nu \rho \sigma}}$.

To perform the geometric optics approximation, we take a background solution of (2.3), $\left\{g_{\mu \nu}, \bar{A}_{\mu}\right\}$, and introduce a vector potential fluctuation $\delta A_{\mu}$ which is then expanded as a product of a slowly varying amplitude and a rapidly varying phase,

$$
\delta A_{\mu}=\left(a_{\mu}+\epsilon b_{\mu}+\ldots\right) \exp \left(\frac{i \vartheta(x)}{\epsilon}\right)
$$

where $\epsilon$ is a small, formal constant introduced to keep track of orders in the expansion. We then derive the equation of motion from the effective action (2.3), evaluate on $g_{\mu \nu}$ and 
$A_{\mu}=\bar{A}_{\mu}+\delta A_{\mu}$ and start expanding, keeping only the terms first order in $\delta A$ and lowest non-trivial order in $\epsilon$, all the while working perturbatively in the effective field theory expansion $\frac{\partial}{m_{e}}$.

The full photon equation of motion is

$$
\begin{aligned}
\nabla^{\nu} F_{\nu \mu}= & 4 e^{2} m_{e}^{-2}\left(a \nabla^{\nu}\left(R F_{\nu \mu}\right)+b \nabla^{\nu}\left(R_{[\nu|\alpha|} F^{\alpha}{ }_{\mu]}\right)+c \nabla^{\nu}\left(R_{\nu \mu \rho \sigma} F^{\rho \sigma}\right)-d \nabla^{\mu}\left(\nabla_{[\mu} \nabla_{|\sigma|} F^{\sigma}{ }_{\nu]}\right)\right) \\
& +8 e^{2} m_{e}^{-4}\left(y \nabla^{\nu}\left(F_{\sigma \tau} F_{\nu}{ }^{\sigma} F_{\mu}^{\tau}+z \nabla^{\nu}\left(F^{2} F_{\nu \mu}\right)\right)\right)
\end{aligned}
$$

and we work in Lorenz gauge

$$
\nabla^{\mu} \delta A_{\mu}=0
$$

Both the gauge condition and equation of motion are expanded in powers of $\epsilon$. In addition, because we truncated the effective action, we are working perturbatively in $m_{e}^{-1}$. Therefore, on the right hand side of (3.4) we may use the zero-th order in $m_{e}^{-1}$ uncorrected black hole solution. For Schwarzschild black holes, only the $c$ term in (3.4) contributes since all other terms are proportional to $\bar{F}_{\mu \nu}, R_{\mu \nu}$ or $R$, all of which are vanishing on the zero-th order solution. The dispersion relation arises at $\mathcal{O}\left(\epsilon^{-2}\right)$, stemming from terms in (3.4) with two derivatives acting on $\delta A$.

Defining $k_{\mu} \equiv \nabla_{\mu} \vartheta$, the leading $\mathcal{O}\left(\epsilon^{-1}\right)$ part of the gauge condition reads

$$
k^{\mu} a_{\mu}=0
$$

which can be used to simplify the $\mathcal{O}\left(\epsilon^{-2}\right)$ part of the equation of motion (3.4) to the form

$$
k^{\nu} k_{\nu}=8 c e^{2} m_{e}^{-2} R_{\mu \rho \nu \sigma} k^{\mu} k^{\nu} f^{\rho} f^{\sigma},
$$

after writing $a_{\mu}=a f_{\mu}$ with $f_{\mu}$ a unit vector, $g^{\mu \nu} f_{\mu} f_{\nu}=1$.

The photon propagation is more naturally phrased in terms of an optical metric $\tilde{g}_{\mu \nu}$ defined by

$$
\tilde{g}^{\mu \nu} \equiv g^{\mu \nu}-8 c e^{2} m_{e}^{-2} R_{\rho \sigma}^{\mu}{ }_{\rho}^{\nu} f^{\rho} f^{\sigma}, \quad \tilde{g}_{\mu \nu} \approx g_{\mu \nu}+8 c e^{2} m_{e}^{-2} R_{\mu \rho \nu \sigma} f^{\rho} f^{\sigma}
$$

Photons are null with respect to this effective metric, $\tilde{g}^{\mu \nu} k_{\mu} k_{\nu}=0$, and follow the geodesics of $\tilde{g}_{\mu \nu}$, not the background metric. ${ }^{16}$ The tangent vector along the photon worldline, $\frac{\mathrm{d} x^{\mu}}{\mathrm{d} \lambda}$, is thus proportional to $\tilde{k}^{\mu}$, defined by

$$
\tilde{k}^{\mu} \equiv \tilde{g}^{\mu \nu} k_{\nu}
$$

not $k^{\mu}$ (as was emphasized recently in [74]).

\footnotetext{
${ }^{16}$ This is easily proven by defining $\tilde{k}^{\mu}=\tilde{g}^{\mu \nu} k_{\nu}=\tilde{g}^{\mu \nu} \nabla_{\nu} \vartheta$ and taking a covariant derivative (with respect to $\left.\tilde{g}_{\mu \nu}\right)$ of the null condition: $0=\frac{1}{2} \tilde{\nabla}_{\alpha}\left(\tilde{g}^{\mu \nu} k_{\mu} k_{\nu}\right)=\tilde{k}^{\nu} \tilde{\nabla}_{\nu} k_{\alpha}$ implying $\tilde{k}^{\nu} \tilde{\nabla}_{\nu} \tilde{k}^{\mu}=0$ which is the standard geodesic equation (we used $\tilde{\nabla}_{[\mu} k_{\nu]}=0$ as $k_{\nu}$ is the gradient of a scalar).
} 

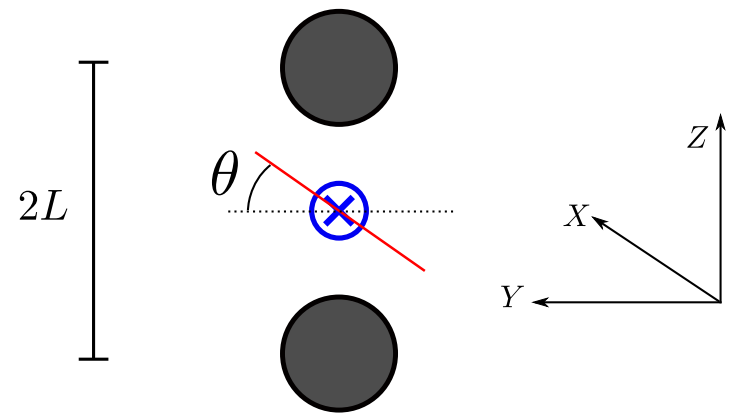

Figure 7. Sketch of the photon traveling into the page. The red line represents the photon polarization vector which makes an angle $\theta$ with the $Z=0$ plane. Photon propagation is maximally timelike if $\theta=0, \pi$ and maximally spacelike if $\theta= \pm \pi / 2(3.11)$.

The interesting question is therefore whether $\tilde{k}^{\mu}$ is spacelike, timelike or null with respect to the background metric $g_{\mu \nu}$, as this is the measure of how different photon propagation in full QED is from naive expectations. At lowest non-trivial order in $m_{e}^{-1}$, this test reads

$$
g_{\mu \nu} \tilde{k}^{\mu} \tilde{k}^{\nu} \approx-8 c e^{2} m_{e}^{-2} R_{\mu \rho \nu \sigma} k^{\mu} f^{\rho} k^{\nu} f^{\sigma}
$$

For our setup in figure 1, we take the photon's polarization vector to make an angle $\theta$ with respect to the positive $Y$ axis, see figure 7, and find:

$$
g_{\mu \nu} \tilde{k}^{\mu} \tilde{k}^{\nu} \approx-\frac{24 c e^{2} L^{2} r_{s} \cos 2 \theta}{m_{e}^{2}\left(L^{2}+X^{2}\right)^{5 / 2}},
$$

where we used (3.2) and took $k^{\mu} \approx(1,1,0,0)+\mathcal{O}\left(r_{s} / \sqrt{L^{2}+X^{2}}\right)$ on the r.h.s. of (3.10). Since $c>0(2.4)$, we see that if the polarization vector lies in the plane of the black hole pair, $\theta= \pm \pi / 2$, then the propagation is maximally spacelike and superluminal, while if the polarization vector is perpendicular to this plane, $\theta=0, \pi$, then the propagation is maximally timelike and subluminal.

In order to analyze the effect on the photon's path in greater detail, we can perturbatively solve for the altered photon geodesic. ${ }^{17}$ The solution for $x^{\mu}(\lambda)$ is conveniently expressed as an expansion about $\bar{x}^{\mu}(\lambda)$, the geodesic whose tangent vector is $\bar{k}^{\mu}$. To lowest non-trivial order,

$$
\bar{x}^{\mu}(\lambda) \approx\left(\lambda+2 r_{s} \ln \left[\lambda / L+\sqrt{1+\lambda^{2} / L^{2}}\right], \lambda, 0,0\right)
$$

\footnotetext{
${ }^{17}$ The easiest way to do this in practice is to solve for $k^{\mu}$ first, translate the result into $\tilde{k}^{\mu}=\tilde{g}^{\mu \nu} g_{\nu \sigma} k^{\sigma}$ and then integrate to find $x^{\mu}(\lambda)$. It is straightforward to demonstrate that this is equivalent to solving $\tilde{k}^{\nu} \tilde{\nabla}_{\nu} \tilde{k}_{\mu}=0$ directly. To solve for $k^{\mu}$, we take a covariant derivative of (3.7) with respect to $g_{\mu \nu}$ to derive a modified geodesic equation: $k^{\nu} \nabla_{\nu} k_{\alpha}=\nabla_{\alpha}\left(4 c e^{2} m_{e}^{-2} R_{\mu \rho \nu \sigma} k^{\mu} k^{\nu} f^{\rho} f^{\sigma}\right)$. Then, $k^{\mu}$ is expanded about a null geodesic of the background metric, $k^{\mu}=\bar{k}^{\mu}+\delta k^{\mu}$ where $\bar{k}^{\mu}$ satisfies $\bar{k}^{\nu} \nabla_{\nu} \bar{k}_{\mu}=0$, and we solve for $\delta k^{\mu}$ perturbatively.
} 
where we took $\lambda=0$ to correspond to a photon at the origin. Writing $x^{\mu}(\lambda)=\bar{x}^{\mu}(\lambda)+$ $\delta x^{\mu}(\lambda)$, we find

$$
\delta x^{\mu}(\lambda) \approx\left(0,-\frac{4 c e^{2} r_{s} \lambda\left(3 L^{2}+2 \lambda^{2}\right)}{m_{e}^{2} L^{2}\left(L^{2}+\lambda^{2}\right)^{3 / 2}} \cos 2 \theta, 0,0\right),
$$

to first order in $c$ and lowest order in $r_{s}$. In (3.13), we've switched to a non-affine parameter in order to simplify the expression and keep $\delta x^{0}(\lambda)=0$ for all $\lambda, \theta$ making the comparison between $x^{\mu}(\lambda)$ and $\bar{x}^{\mu}(\lambda)$ more straightforward.

By calculating $\delta x^{\mu}(\lambda)$ we are effectively comparing the flight of a non-minimally coupled photon to the flight of a minimally coupled "test" photon on the same background in order to understand how much the QED photon's propagation differs from that of a "normal" photon. We denote the non-minimally coupled photon by $\gamma_{\mathrm{QED}}$ and the minimally coupled photon by $\gamma_{\min }$ with the former's motion dictated by $(2.3)$, while the latter's motion would be described by only a Maxwell term. We will often refer to $\gamma_{\min }$, but if one would rather avoid referring to degrees of freedom not explicitly included in the theory, the entire analysis can be rephrased as a comparison between the strictly luminal QED photon $(\theta=\pi / 4)$ and the other possible polarizations of $\gamma_{\mathrm{QED}}$.

This comparison between photons on the same background avoids the complications which would arise if we were to, for example, compare the QED photon's trajectory in a black hole background to the trajectory of a null path in Minkowski space. Difficulties even arise in attempting to contrast the trajectory of a minimally coupled photon, $\gamma_{\min }$, in Schwarzschild to a flat space photon as the logarithmic Shapiro time delay term in $\bar{x}^{\mu}(3.12)$ causes the Schwarzschild photon to fall behind its flat space counterpart by a diverging amount $\propto r_{s} \ln \lambda$. See [75, 76] for discussions of related topics. By comparing trajectories in the same background, we sidestep such issues.

From (3.13), we can immediately compare the paths of the different photons. If $\gamma_{\mathrm{QED}}$ and $\gamma_{\text {min }}$ were to race from directly between the black holes out to infinity, the asymptotic difference between the two paths is

$$
\Delta X \approx \lim _{\lambda \rightarrow \infty} \delta x^{1}(\lambda)=-m_{e}^{-1}\left(\frac{8 c e^{2} r_{s}}{m_{e} L^{2}}\right) \cos 2 \theta .
$$

Thus, a maximally subluminal QED photon would lose to $\gamma_{\min }$ by a distance $m_{e}^{-1}\left(\frac{8 c e^{2} r_{s}}{m_{e} L^{2}}\right)$ which in turn would lose to the maximally superluminal QED photon by the same amount, in agreement with our estimate (1.4). See figure 8.

This distance, for the values $L \gtrsim r_{s}, L \gg m_{e}^{-1}, e \sim \mathcal{O}(1)$, is $\ll m_{e}^{-1}$, well outside the regime of validity of the EFT.

\section{Building up QED superluminality}

In this section we attempt to build up the QED superluminality with the goal of achieving $\Delta X>m_{e}^{-1}$. We start by discussing two simple attempts which can quickly be shown to fail. Afterwards, we introduce the main amplifying scenario considered in this paper: a ladder of approximately extremal Reissner-Nordstrom (RN) black holes. 


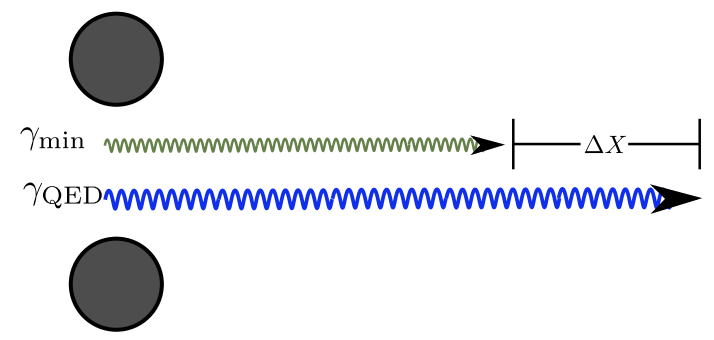

Figure 8. Exaggerated sketch of the race between a maximally superluminal photon, $\gamma_{\mathrm{QED}}$ with $\theta= \pm \pi / 2$, and a minimally coupled photon, $\gamma_{\min }$. The QED photon wins by $\Delta X \approx m_{e}^{-1}\left(\frac{8 c e^{2} r_{s}}{m_{e} L^{2}}\right)$.

\subsection{Simple attempts: large $N_{f}$, small $r_{s}$ and photon orbits}

Examining the expression for the QED distance advance, $\Delta X \approx m_{e}^{-1}\left(\frac{8 c e^{2} r_{s}}{m_{e} L^{2}}\right)$, a few methods of amplification immediately come to mind. Start by noting that the advance is bounded by taking the $L \rightarrow r_{s}$ limit of $\Delta X$ :

$$
\Delta X \leq m_{e}^{-1}\left(\frac{8 c e^{2}}{m_{e} r_{s}}\right)
$$

corresponding to skipping the photon off of the $\mathrm{BH}$ horizon. We are interested in making $\left(\frac{8 c e^{2}}{m_{e} r_{s}}\right) \gg 1$ and the two basic strategies are to either make the numerator large or the denominator small.

The numerator can be made large by considering a new version of the problem where we work with $N_{f}$ flavors of electrons, instead of just one. In this case, the distance advance formula is changed to

$$
\Delta X \approx m_{e}^{-1}\left(\frac{8 c N_{f} e^{2} r_{s}}{m_{e} L^{2}}\right)
$$

and the prescription is to take $N_{f} e^{2} \gg 1$. However, this limit cannot be taken while retaining perturbative control of the theory. The quantity $N_{f} e^{2}$ is the 't Hooft coupling (with $N_{f}$ the number of flavors, rather than the rank of the gauge group) and the oneloop vacuum polarization correction to the photon propagator is $\propto N_{f} e^{2}$. Large 't Hooft coupling means non-perturbative photon dynamics which implies that we can't trust the approximations we have made in deriving and truncating the effective action.

The denominator of (4.1) can be made small by studying tiny black holes, those for which $r_{s} m_{e} \ll 1$. However, such minuscule black holes are well outside of the validity of the EFT. Heuristically, they are objects of size much smaller than the cutoff of the EFT, $r_{s} \ll m_{e}^{-1}$, and hence are not describable. More quantitatively, the bound (4.1) comes from shooting the photons quite close to the horizon of the black hole where the curvature is of order $R_{\mu \nu \rho \sigma} \sim 1 / r_{s}^{2}$, meaning that $R_{\mu \nu \rho \sigma} / m_{e}^{2} \sim 1 /\left(m_{e} r_{s}\right)^{2} \gg 1$ and hence our truncation of the EFT (2.3) is invalid for this setup, as we've dropped terms which are higher order in $R_{\mu \nu \rho \sigma} / m_{e}^{2}$ that are in no way suppressed relative to the terms we've kept.

Finally, there is no obvious restriction on building up an integrated macroscopic distance advance by choosing the photon to orbit a large black hole for many cycles. However, 


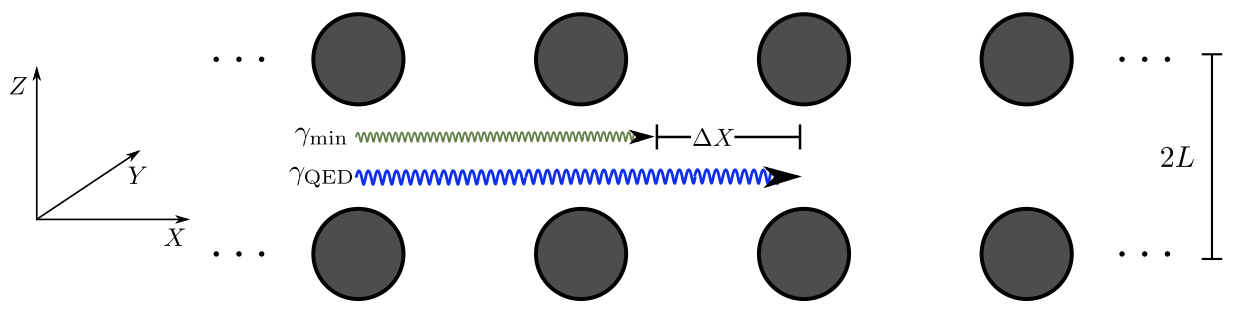

Figure 9. Racing $\gamma_{\min }$ and $\gamma_{\mathrm{QED}}$ down a ladder of black holes.

this setup does not permit us to send signals between asymptotic observers any faster than if there were no black hole at all, so it is not the type of sharp asymptotic observable we are interested in.

\subsection{A ladder of black holes: large $N_{\mathrm{BH}}$}

A more fruitful direction to push is the limit of many black holes. We consider building a ladder of $N_{\mathrm{BH}}$ black holes, arranged in pairs with each pair constituting a rung of the ladder, and racing $\gamma_{\text {QED }}$ against $\gamma_{\text {min }}$ down the middle of the ladder, see figure 9 .

If we could construct a ladder of arbitrary length, we could clearly make the distance advance as large as we wish. We cannot, however, as the multi-black hole solution is not generally static since the black holes mutually attract. Initially placing the black holes at vertical separation $2 L$, the photon race until the separation becomes $\mathcal{O}\left(r_{s}\right)$, at which point the black holes start to merge and the ladder collapses.

Analytic control of the race is lost when the ladder coalesces, so we should attempt to prolong the lifespan of the setup. One way to accomplish this is to use identical extremal Reissner-Nordstrom black holes. Famously, the sum of many stationary, extremal RN black holes is also an exact, stationary solution to pure Einstein-Maxwell theory [77-79], because the electromagnetic repulsion perfectly balances the gravitational attraction. Our ladder is thus perfectly stable in such a theory.

However, since we are working with full QED, not just Einstein-Maxwell, these Majumdar-Papapetrou spacetimes are only approximate solutions and the additional operators in the EFT (2.3) introduce new effects. Further, they are only classical solutions of Einstein-Maxwell theory: graviton and photon loops must also be accounted for. We analyze the new effects in the following sections and determine whether they destabilize the ladder quickly enough to avoid macroscopic superluminality.

\section{Black hole ladder analysis}

Here we study the ladder of approximately extremal Reissner-Nordstrom black holes. First, we recall the exact black hole solutions of pure Einstein-Maxwell theory and their relevant properties. Next, we discuss how to calculate the perturbative corrections to these solutions, due to the electron-induced operators in the EFT (2.3). Effects of photon and graviton loops are then discussed separately, as their treatment is slightly more subtle. Finally, we bound the distance advance acquired by $\gamma_{\mathrm{QED}}$ in this idealized scenario. 


\subsection{Einstein-Maxwell background}

The pure Einstein-Maxwell action is:

$$
S_{\mathrm{EM}}=\int \mathrm{d}^{4} x \sqrt{-g}\left[\frac{M_{p}^{2}}{2} R-\frac{1}{4 e^{2}} F_{\mu \nu}^{2}\right]-M \int \mathrm{d} \tau+\frac{Q}{e} \int \mathrm{d} x^{\mu} A_{\mu},
$$

where we've explicitly included the source terms for a single black hole of mass $M$ and charge $Q$ ( $\tau$ parametrizes the worldline of the charged source). The background equations of motion from (5.1) read

$$
M_{p}^{2} G_{\mu \nu}=\frac{1}{e^{2}}\left[F_{\mu}{ }^{\alpha} F_{\nu \alpha}-\frac{1}{4} g_{\mu \nu} F^{2}\right], \quad \nabla^{\nu} F_{\mu \nu}=0,
$$

and are satisfied by the Reissner-Nordstrom solution

$$
\begin{aligned}
\mathrm{d} s^{2} & =-\Delta(r) \mathrm{d} t^{2}+\Delta(r)^{-1} \mathrm{~d} r^{2}+r^{2} \mathrm{~d} \Omega_{2}^{2} & & \\
\Delta(r) & =1-\frac{r_{s}}{r}+\frac{r_{q}^{2}}{4 r^{2}}, & F^{t r} & =\frac{e M_{p}}{\sqrt{2}} \frac{r_{q}}{r^{2}} \\
r_{s} & \equiv \frac{M}{4 \pi M_{p}^{2}}, & r_{q} & \equiv \frac{Q}{\pi \sqrt{8} M_{p}},
\end{aligned}
$$

with all other components of $F_{\mu \nu}$ vanishing or related to (5.3) by symmetries. The extremal black hole arises in the limit $r_{q} \rightarrow r_{s}$, at which point $\Delta(r)$ factorizes: $\Delta(r)=\left(1-\frac{r_{s}}{2 r}\right)^{2}$.

In isotropic coordinates, $x^{\mu}=(t, X, Y, Z)$ with $X^{2}+Y^{2}+Z^{2}=\left(r-r_{s} / 2\right)^{2},(5.3)$ can be generalized to the exact Einstein-Maxwell solution containing $N_{\mathrm{BH}}$ equal mass, extremal black holes at locations $\vec{X}_{i}[79]$ :

$$
\mathrm{d} s^{2}=-U^{-2} \mathrm{~d} t^{2}+U^{2} \mathrm{~d} \vec{X}^{2}, \quad A_{0}=e M_{p} \sqrt{2} U^{-1}, \quad U \equiv 1+\sum_{i=1}^{N_{\mathrm{BH}}} \frac{r_{s}}{2\left|\vec{X}-\vec{X}_{i}\right|} .
$$

\subsection{Quantum corrections}

Since we are not working with pure Einstein-Maxwell, but rather the EFT (2.3), the configurations of the previous sections are only approximate solutions. There are multiple ways of calculating how the new EFT operators in (2.3) affect the configuration. We find it most transparent to phrase the calculation in terms of Feynman diagrams for perturbatively solving the equations of motion [80]. The leading corrections to the single extremal RN solution are calculated and then multiple copies are superimposed to find the approximate multi-black hole metric. Forces between nearby black holes are then calculated using the geodesic equation and the resulting dynamics are calculated in the Newtonian approximation.

Each of these steps involves approximations, but the errors are expected to be small in each case:

- Black hole solutions cannot generically be added together to form new solutions, due to the non-linearity of GR. However, as long as the separation between black holes is much larger than their respective horizon sizes, the composite metric should serve as a good approximation. Our setup falls within this regime. 
- Placing the black holes at some initial separation, we find the Newtonian potential between a single pair of perturbed black holes and calculate how quickly they come within a distance $\sim r_{s}$ of each other. This estimate serves as an upper bound for the lifetime of the entire ladder, which is all we'll need. Using a Newtonian description is only valid for weak gravitational forces and velocities much smaller than $c$. Our setup falls within this regime (as we'll verify).

- In practice, we've calculated contributions to $\left\langle h_{\mu \nu}\right\rangle$ using time-ordered Feynman rules. Strictly speaking, these "in-out" matrix elements are only equivalent to expectation values if the system is in equilibrium. In order to calculate true expectation values and capture non-equilibrium effects such as Hawking radiation (see [81] for a recent such study), one would instead need to use the full Schwinger-Keldysh or "in-in" formalism [82, 83]. However, because the black holes are nearly extremal, their evaporation rate is minuscule and we expect such effects to be negligible.

- Photon and graviton loops generate subtle corrections to the metric, which are not immediately interpretable as unambiguous corrections to the Newtonian potential. They require a more careful treatment, as is discussed in section 5.2.4.

\subsubsection{Feynman diagram estimation: $\mathrm{RN}$ black holes}

Finding black hole solutions via diagrams has a long history. Duff pioneered the subject, both reproducing the usual, classical Schwarzschild solution [84] and finding the leading quantum corrections to the metric [85] from graviton loops (though, this latter subject turns out to be surprisingly subtle, see section 5.2.4). Though diagrams can be used to find the exact perturbative corrections with all numerical coefficients determined, the primary utility of Feynman diagrams for the present purpose is their efficiency in estimating, comparing and organizing competing corrections to $h_{\mu \nu}$ and $A_{\mu}$.

Consider building up the generic RN solution via Feynman diagrams. The schematic form of the Einstein-Maxwell action, with source terms included,

$$
\mathcal{L} \sim l_{p}^{-2} h^{n}(\partial h)^{2}+\frac{1}{e^{2}} h^{n}(\partial A)^{2}+r_{s} l_{p}^{-2} h \delta^{3}(r)+\frac{r_{q} l_{p}^{-1}}{e} A \delta^{3}(r),
$$

is sufficient for our purposes. We found it convenient to write all quantities in terms of length scales, $l_{p}=M_{p}^{-1}, r_{s} \sim M l_{p}^{2}$ and $r_{q} \sim Q l_{p}$. The derivation of the Feynman rules is standard and we'll only need their schematic form:

- All diagrams are drawn as sources feeding into $\left\langle h_{\mu \nu}\right\rangle$ or $\left\langle A_{\mu}\right\rangle$ from right to left.

- ceeeeeee is a graviton line, appearing with a factor $\sim l_{p}^{2}$.

- munu is a photon line, appearing with a factor $\sim e^{2}$.

- Any line whose right end is bare has a source attached to that end.

- If ceeeeeee has a source attached, it gets another factor of $\sim r_{s} l_{p}^{-2}$. 
(a)

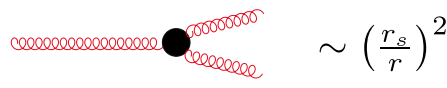

(c)

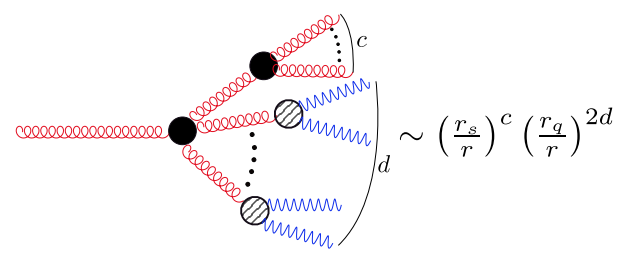

(b)<smiles>[GeH3]</smiles>

(d)



Figure 10. Some examples of Feynman diagrams for the Reissner-Nordstrom metric solution. Diagrams $(a)$ and $(b)$ are the leading GR correction and charge contribution. Diagrams $(c)$ and $(d)$ are complicated diagrams whose sizes are easily estimated.

- If munu has a source attached, it gets another factor of $\sim r_{q} l_{p}^{-1} e^{-1}$.

- is an Einstein-Hilbert vertex, appearing with a factor $\sim l_{p}^{-2}$.

- $\bigotimes$ is a Maxwell vertex, appearing with a factor $\sim e^{-2}$.

- Overall dimensions are fixed by inserting factors of $r$.

We will refer to any line whose right end is bare as "external." These rules allow for fast and easy estimations of the various contributions to the solution.

For instance, the linear solution for the metric corresponds to a single graviton line: ceeeeeee. The estimation of this diagram is simple. Every graviton line comes with a factor of $\sim l_{p}^{2}$ and since the right end of the single line is bare, there's also a single factor of the source $\sim r_{s} l_{p}^{-2}$. Feynman rules and dimensional analysis quickly yield the estimate of the standard Newtonian potential,

$$
\left\langle h_{\mu \nu}\right\rangle=\text { ceeeeeee } \approx \frac{1}{r} \times l_{p}^{2} \times r_{s} l_{p}^{-2}=\frac{r_{s}}{r} .
$$

We similarly estimate that the linearized photon solution is

$$
\left\langle A_{\mu}\right\rangle=\operatorname{mun} \approx \frac{1}{r} \frac{e r_{q}}{l_{p}} .
$$

The first GR correction and the leading contribution of charge to the metric arise from cubic vertices, as shown in figure $10(a)$ and $(b)$. Both are easily estimated and are found to be of the correct form, as can be seen by comparing to the full solution (5.3). It's also easy to estimate the sizes of more complicated diagrams, see figure $10(c)$ and $(d)$.

This diagrammatic language greatly helps to organize the calculation. In the limiting case of finding the classical, extremal RN solution via Feynman diagrams, the organization is fairly trivial: there's only one scale $r_{s}$ entering the metric solution and a diagram with $n$ external legs and arbitrarily many vertices gives a contribution of size $\sim\left(r_{s} / r\right)^{n}$ to $\left\langle h_{\mu \nu}\right\rangle$. However, when we consider both classical and quantum diagrams built using the 
full EFT (2.3) several more scales and factors appear $\left(e, m_{e}\right.$ and $\left.l_{p}\right)$ and diagrams become an invaluable organizational tool.

Finally, note that important physics is clearly expressed through these estimates: diagrams tell us the scale at which physics qualitatively changes due to non-linearities and the breakdown of perturbation theory. For instance, the linear Schwarzschild BH solution is $\left\langle h_{\mu \nu}\right\rangle \sim r_{s} / r$ while the non-linear corrections are all of size $\sim\left(r_{s} / r\right)^{n}$. These GR corrections are therefore small for $r \gtrsim r_{s}$ and important for $r \lesssim r_{s}$, at which point perturbation theory breaks down and we need to find the full non-linear solution for the metric (5.3). A similar analysis for generic RN black holes demonstrates that non-linearities are important at the whichever scale is largest among $r_{s}, r_{q} . r_{s} \gg r_{q}$ corresponds to horizon formation, $r_{s} \ll r_{q}$ corresponds to a naked singularity and $r_{s} \sim r_{q}$ is a special neighborhood containing the extremal black hole. Generically, physics changes qualitatively when non-linearities become important and Feynman diagrams provide a quick way of determining where these interesting non-linear scales lie.

\subsubsection{Tree diagrams for $\left\langle h_{\mu \nu}\right\rangle$}

Now we estimate the sizes of the various metric corrections coming from the new EFT operators in (2.3). Since the background forces cancel for the approximately extremal $\mathrm{RN}$ black holes we are considering, these electron induced corrections provide the leading gravitational forces which destabilize the ladder setup. Summing up tree diagrams is equivalent to taking the equations of motion derived from (2.3) and solving perturbatively. As we'll see, this alone is not sufficient and misses important contributions to the solution.

The schematic Lagrangian now reads:

$$
\mathcal{L} \sim l_{p}^{-2} h^{n}(\partial h)^{2}-\frac{1}{4 e^{2}} h^{n}(\partial A)^{2}+r_{e}^{2} h^{n} \partial^{2} h(\partial A)^{2}+r_{e}^{4} h^{n}(\partial A)^{4}-r_{s} l_{p}^{-2} h \delta^{3}(r)+\frac{r_{q} l_{p}^{-1}}{e} A \delta^{3}(r)
$$

where we introduced the length scale $r_{e} \equiv m_{e}^{-1}$. The third term in (5.8) corresponds to all of the $\sim m_{e}^{-2} R F F$ operators, the fourth corresponds to the $\sim m_{e}^{-4} F^{4}$ operators and we neglected the $d$ operator $\sim m_{e}^{-2}(\partial F)^{2}$ as it's redundant and only ends up providing subleading corrections. A single new Feynman rule is sufficient for estimating the sizes of the new diagrams:

- $\mathbb{H}_{\text {is }}$ an electron induced vertex.

- If $\mathbb{W}_{\text {has }}$ two photon lines attached, it corresponds to the third term in (5.8) and appears with a factor $\sim r_{e}^{2}$.

- If $\mathbb{T}^{\mathrm{S}}$ has four photon lines attached, it corresponds to the fourth term in (5.8) and appears with a factor $\sim r_{e}^{4}$.

The simplest EFT corrections to the metric are shown in figure 11. Easy estimates demonstrate that a diagram utilizing both an insertion of the Maxwell term and an $m_{e}^{-4} F^{4}$ operator and a diagram using only a single $\sim m_{e}^{-4} F^{4}$ insertion are of the same 




$(a)$



(b)

Figure 11. The simplest tree-level EFT corrections to the metric.

order. The former corresponds to finding the correction to $A_{\mu}$ from the $\sim F^{4} / m_{e}^{4}$ operators and feeding the result into the Einstein-Maxwell $T_{\mu \nu}$ to find how it affects the metric. This correction is just as important as the $\sim F^{4} / m_{e}^{4}$ operators' direct contribution to the metric (the lower diagram in figure 11). Comparing the two diagrams in figure 11, one finds that the $(a)$ dominates for $r \lesssim r_{s}\left(\frac{e r_{e}}{l_{p}}\right)$ and $(b)$ dominates for $r \gtrsim r_{s}\left(\frac{e r_{e}}{l_{p}}\right)$. The form of the gravitational force law changes depending on how separated the black holes are.

The factor $e r_{e} / l_{p}=e M_{p} / m_{e}$ arises often in the calculation and is exactly the quantity that the Weak Gravity Conjecture states should be larger than unity in any theory which can be UV completed into a consistent theory including quantum gravity [71]. The Standard Model electron satisfies this bound easily, $e r_{e} / l_{p} \sim 10^{22}$, and (unless specified otherwise) we proceed assuming that our theory also satisfies this bound, as we wish to stay as close to real world QED as possible.

Finally, we can double check that our calculation is within the validity of the EFT. The existence of electrons shouldn't produce drastic changes to the background solutions, otherwise we are not in the regime where the EFT is valid. This means that all diagrams using EFT vertices should be small compared to the background solution in the regime of interest, $r \gtrsim r_{s}$. Equivalently, this implies that any new non-linear scales induced by the new EFT operators should be smaller than $\sim r_{s}$, so that GR's non-linearities always become important first.

These properties do not hold for all black holes: there is a minimal size black hole below which the EFT description breaks down. In order to see this, consider evaluating the two diagrams in figure 11 just outside the horizon, $r \sim r_{s}$ where the linear solution and all Einstein-Maxwell corrections are starting to become $\mathcal{O}(1)$. If electrons aren't very important, then both diagrams should be $\ll \mathcal{O}(1)$ in this regime. Figures $11(a)$ and $(b)$ satisfy this condition only if $r_{s} \gg e r_{e}\left(e r_{e} / l_{p}\right)$ and $r_{s} \gg l_{p}\left(e r_{e} / l_{p}\right)$, respectively, and the first constraint is strongest, due to the WGC assumption.

More stringent bounds come from considering different diagrams. It turns out that the strongest constraints come from the diagram with a single insertion of an operator $\sim r_{e}^{4 n} h(\partial A)^{2(n+1)}$ with $n \rightarrow \infty$. The diagram gives

$$
\left\langle h_{\mu \nu}\right\rangle \sim\left(\frac{e r_{e}}{l_{p}}\right)^{n+1}\left(\frac{r_{e}}{r}\right)^{n-1}\left(\frac{r_{s}}{r}\right)^{2(n+1)}\left(\frac{l_{p}}{r}\right)^{2}
$$


and $\left\langle h_{\mu \nu}\right\rangle \ll \mathcal{O}(1)$ at $r \sim r_{s}$ iff $r_{s} \gtrsim r_{e}\left(\frac{e r_{e}}{l_{p}}\right)$. If the bound is violated, an infinite tower of operators generate important corrections to the solution. Again, this analysis is equivalent to identifying the distance scale at which EFT non-linearities become important and then demanding that this scale be smaller than $r_{s}$.

The bound $r_{s} \gtrsim r_{e}\left(\frac{e r_{e}}{l_{p}}\right)$ is nothing but the statement that our EFT description is only valid for field strengths obeying $F^{2} / m_{e}^{2} \ll 1$. The strongest field strengths we probe are of order

$$
\left.\left.F_{\mu \nu}\right|_{r=r_{s}} \sim \partial A\right|_{r=r_{s}} \sim \frac{e}{r_{s} l_{p}}
$$

which is smaller than $m_{e}^{2}$ only if ${ }^{18} r_{s} \gtrsim r_{e}\left(\frac{e r_{e}}{l_{p}}\right)$, which is the condition we found through diagrams. Physically, we expect rampant $e^{+}, e^{-}$Schwinger pair production when this condition is violated, ${ }^{19}$ in agreement with the scale found in the detailed pair production analysis of [86].

The rough estimates given above are fully realized in the precise results of the actual calculation [87]. A similar analysis for the vector potential solution is straightforward and yields the same conclusions. After finding the leading perturbative corrections, we can simply read off the electron corrections to $h_{00}$ to find the gravitational potential induced by electrons, and similarly for the zero component of the vector potential.

\subsection{3 $\left\langle h_{\mu \nu}\right\rangle$ from photon and graviton loops}

The tree diagrams of the previous section miss an important effect: the contribution of graviton and photon loops. Not only should these be included for consistency, they also generate the dominant corrections at long distances and qualitatively change the dynamics. These corrections would be missed entirely if one simply tried to find the metric via perturbatively solving the EFT equations of motion derived from (2.3). Instead, they are captured by the 1PI effective action, discussed later. The use of Feynman diagrams makes it particularly clear that these corrections need to be included and quickly singles out the regime where they dominate.

Physically, it's entirely reasonable that loops of photons and gravitons should compete with the effects of the new EFT operators and that they should dominate at long distances. Recall that the generated EFT operators all arose via electron loops, as in figure 5, and hence the tree diagrams considered in the previous section correspond to loop diagrams in the full theory. They represent quantum effects. Since photon and graviton loops represent the quantum corrections from massless particles, their effects should be very long ranged, dominating the corrections far from the source, while electron loop effects dominate at shorter distances.

\footnotetext{
${ }^{18}$ Funny numerology occurs when this bound is evaluated for the Standard Model. In terms of the $\mathrm{BH}$ mass $\left(M_{\mathrm{BH}}\right)$ and solar masses $\left(M_{\odot}\right)$, a few fundamental numbers $\left(e, M_{p}\right.$ and $\left.m_{e}\right)$ combine to yield the condition $M_{\mathrm{BH}} \gtrsim 10^{5} M_{\odot}$, as pointed out in [86], roughly corresponding to the lower mass range of real world supermassive black holes.

${ }^{19}$ For a generic RN BH, a similar analysis gives the condition $r_{s}^{2} \gg r_{e} r_{q}\left(\frac{e r_{e}}{l_{p}}\right)$.
} 


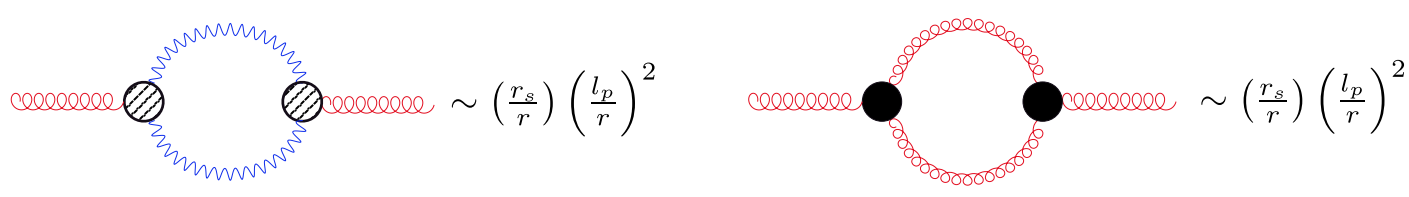

Figure 12. Typical loop corrections to the metric from gravitons and photons. Here, and in following figures, we leave the necessary ghost diagrams implicit.

Typical loops needed for the calculation are shown in figure 12. The full calculation of graviton and photon loops is fairly painful, due to the plethora of indices [88-90]. Fortunately, our Feynman rules for approximating diagrams faithfully reproduce the size of these corrections to the metric, first calculated by Duff [85]. Very closely related (but not entirely equivalent) ideas were later stated in modern EFT language by Donoghue ${ }^{20}$ [95].

The estimate for the correction follows from figure 12: $\delta h_{\mu \nu} \sim \frac{r_{s}}{r}\left(\frac{l_{p}}{r}\right)^{2}$. Comparing these loop corrections to the tree diagrams of the previous section, figure $11(a)$ and $(b)$, we find that light loops dominate the metric corrections at distances $r \gtrsim r_{s}\left(\frac{e r_{e}}{l_{p}}\right)^{2}$.

\subsubsection{Subtleties of gauge loops}

Unfortunately, turning these gauge loop diagrams into a potential is not so straightforward a process. We can't simply find $\delta h_{00}$ and take this to be the potential because the 1PI action for GR is gauge dependent, which makes the correction $\delta h_{\mu \nu}$ ambiguous.

Starting from the GR action with a point source,

$$
S=\int \mathrm{d}^{4} x \sqrt{-g} \frac{M_{p}^{2}}{2} R-M \int \mathrm{d} \tau,
$$

we can calculate the $1 \mathrm{PI}$ action $\Gamma\left[\bar{g}_{\mu \nu}\right]$ via the background field method (BFM) [96] by expanding the Einstein-Hilbert action about $g_{\mu \nu}=\bar{g}_{\mu \nu}+\delta g_{\mu \nu}$ and integrating over all 1PI graphs where only $\delta g_{\mu \nu}$ propagates in loops,

$$
\exp i \Gamma[\bar{g}]=\int_{1 \mathrm{PI}} \mathcal{D} \delta g_{\mu \nu} \exp i S\left[\bar{g}_{\mu \nu}+\delta g_{\mu \nu}\right] .
$$

Actually performing the calculation (5.12) requires gauge fixing for $\delta g_{\mu \nu}$ and we can ensure that $\Gamma\left[\bar{g}_{\mu \nu}\right]$ is built from diffeomorphism invariant operators by making a clever choice of gauge fixing functional [88], for instance $G_{\mu}=\bar{\nabla}^{\nu} \delta g_{\nu \mu}-\frac{1}{2} \bar{\nabla}_{\mu} \delta g^{\nu}{ }_{\nu}$. A gauge fixing term $\mathcal{L}_{\text {gf }}=-\frac{1}{2 \xi} G_{\mu} G^{\mu}$ is then added to the action (along with the associated ghost terms) where $\xi$ is an arbitrary parameter. Performing the necessary integrals, the one-loop 1PI action contains the following non-analytic operators [97]

$$
\begin{aligned}
\Gamma\left[\bar{g}_{\mu \nu}\right] \supset & \int \mathrm{d}^{4} x \sqrt{-\bar{g}}\left(c_{1} \bar{R} \log \left(-\square / \mu^{2}\right) \bar{R}+c_{2} \bar{R}^{\mu \nu} \log \left(-\square / \mu^{2}\right) \bar{R}_{\mu \nu}+c_{3} \bar{R}^{\mu \nu \rho \sigma} \log \left(-\square / \mu^{2}\right) \bar{R}_{\mu \nu \rho \sigma}\right) \\
& -c_{4} \frac{M}{M_{p}^{2}} \int \mathrm{d} \tau \log \left(-\square / \mu^{2}\right) \bar{R}-c_{5} \frac{M}{M_{p}^{2}} \int \mathrm{d} \tau \log \left(-\square / \mu^{2}\right) \bar{R}_{\mu \nu} \frac{\mathrm{d} x^{\mu}}{\mathrm{d} \tau} \frac{\mathrm{d} x^{\nu}}{\mathrm{d} \tau},
\end{aligned}
$$

\footnotetext{
${ }^{20}$ Many, many authors have calculated the correction using a variety of methods. See, for instance, [9193] and [94] for a review. Not all results agree in their precise numerical coefficients, but all find the same order of magnitude as figure 12 .
} 
with some calculable coefficients $c_{i}$. The three new operators in the first line correspond to the vacuum polarization diagrams in figure 12, along with similar diagrams with more external legs. The new operators in the final line come from diagrams using insertions of vertices from the point source term.

Using (5.13), we can calculate $\left\langle\delta h_{\mu \nu}\right\rangle$ in a precise manner: expand $\bar{g}_{\mu \nu}$ about flat space, add a new gauge fixing term to make the propagator invertible and compute tree diagrams using the terms in the second line of (5.13) as the source terms. This is essentially the method used by Duff [85], though the matter corrections $c_{3}, c_{4}$ were neglected there.

The problem, then, is that many of the $c_{i}$ 's in (5.13) depend on the choice of the gauge fixing parameter $\xi$ used to fix the background fluctuation in (5.12). The $\xi$ dependence of the $c_{i}$ 's then feeds into the metric, which also ends up being $\xi$-dependent. While the $\xi$-dependence cancels out of the one-loop, BFM result for $\Gamma\left[\bar{g}_{\mu \nu}\right]$ in Yang-Mills theories, the analogue statement is not true in GR, a property ascribed to the non-renormalizable nature of GR in [98]. The background gauge fixing is logically distinct from the gauge fixing required when using $\Gamma\left[\bar{g}_{\mu \nu}\right]$ to find $\left\langle\delta h_{\mu \nu}\right\rangle$ and represents a true ambiguity. For instance, the value of the Ricci scalar induced via the one-loop corrections in (5.13) depend on $\xi$, but not on the parameter used in gauge fixing $\Gamma\left[\bar{g}_{\mu \nu}\right]$ to compute the necessary tree diagrams.

This is a general property of the effective action for theories with gauge fields; see, for instance, [99-102]. The field profiles which extremize the 1PI effective action are generically gauge dependent, since the form of the 1PI action is itself gauge dependent. Instead of finding the metric, one must use $\Gamma\left[\bar{g}_{\mu \nu}\right]$ to calculate physical quantities such as $S$-matrix elements [103, 104] or modified geodesic equations [97] which account for the non-minimal matter coupling in (5.13), each of which yield $\xi$-independent predictions.

Despite these subtleties in turning the diagrams of figure 12 into precise potentials, the figures and power counting rules constitute a good mnemonic for the calculation: the cor-

rection of the potential due to massless loops is $\delta V \sim \frac{r_{s}}{r}\left(\frac{l_{p}}{r}\right)^{2}[11,92,95,97,103,104]$. Therefore, we continue to use the diagrams of figure 12 as a representation of the effect. The exact one-loop potential is calculated in appendix $\mathrm{C}$ by combining the results of $[103,105,106]$.

\subsubsection{Combining all effects}

Combining the results of the previous two sections, along with the results of the vector potential estimates, we find that the calculation breaks up into three regions where different effects dominate, see figure 13 .

If we were to place another, identical black hole in the spacetime, we'd find that the form of the force law depends on the separation: there are three distinct behaviors, depending on which region of figure 13 we place the second black hole. However, no matter where we place the second black hole, it is found that the corrections generate an attraction between the black holes. Not all of the individual diagrams in figure 13 generate an attractive perturbative correction, but when all corrections are summed up with their precise signs and coefficients, everything works out such that attraction persists at all scales. 


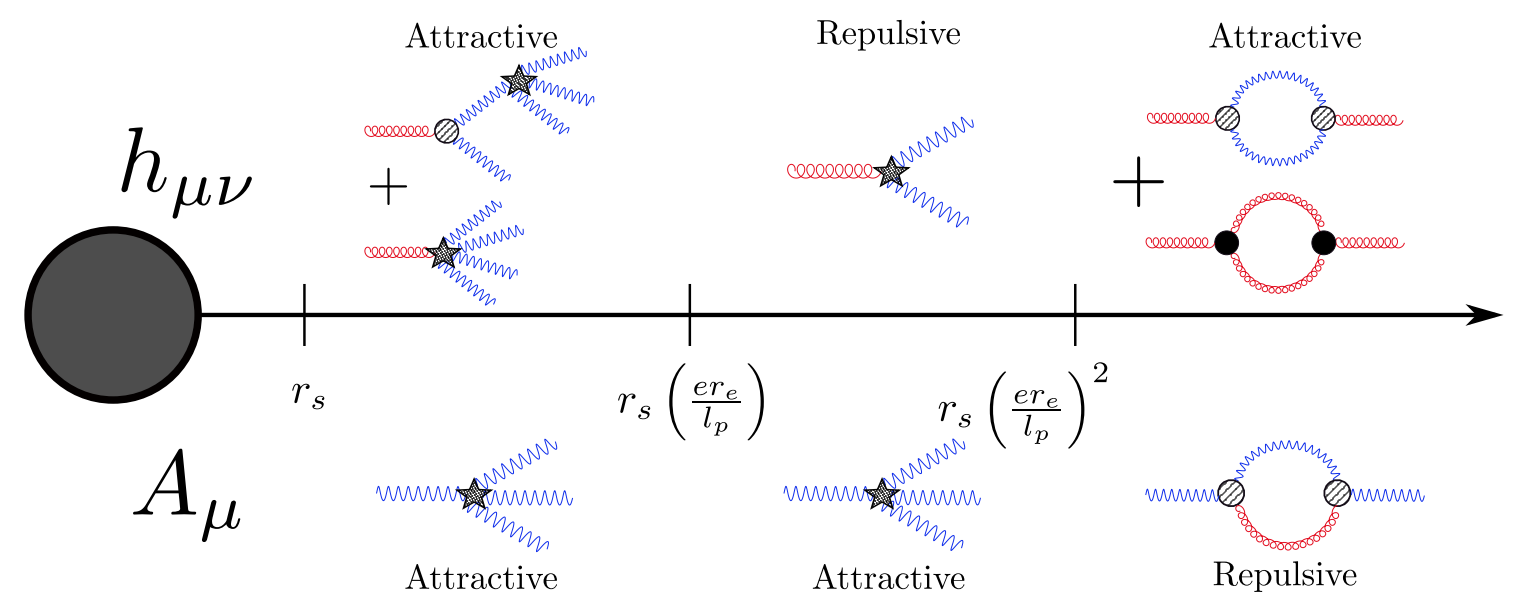

Figure 13. Dominant metric and vector potential corrections at different distances from the black hole. We indicate whether each diagram corresponds to an attractive or repulsive force between this black hole and a second, identical one, which we imagine is placed in the indicated region.

The electron induced effects can be accurately captured as perturbative corrections to the metric and field strength tensor; there are no subtle gauge issues here. Writing the full solution for the metric and vector potential as $g_{\mu \nu}=\bar{g}_{\mu \nu}+\delta g_{\mu \nu}$ and $F_{\mu \nu}=\bar{F}_{\mu \nu}+\delta F_{\mu \nu}$ with $\bar{g}_{\mu \nu}, \bar{F}_{\mu \nu}$ the classical, extremal RN solution of (5.3), it is found [87] that electrons induce the corrections:

$$
\begin{aligned}
\delta g_{t t} & =(c-2 a)\left(\frac{e r_{e}}{l_{p}}\right)^{2}\left(\frac{r_{s}}{r}\right)^{2}\left(\frac{l_{p}}{r}\right)^{2}+\frac{(y+2 z)}{10}\left(\frac{e r_{e}}{l_{p}}\right)^{4}\left(\frac{r_{s}}{r}\right)^{4}\left(\frac{l_{p}}{r}\right)^{2} \\
\delta g_{r r} & =(8 a+3 b+4 c)\left(\frac{e r_{e}}{l_{p}}\right)^{2}\left(\frac{r_{s}}{r}\right)^{2}\left(\frac{l_{p}}{r}\right)^{2}+\frac{(y+2 z)}{10}\left(\frac{e r_{e}}{l_{p}}\right)^{4}\left(\frac{r_{s}}{r}\right)^{4}\left(\frac{l_{p}}{r}\right)^{2} \\
\delta F^{t r} & =\frac{e}{r l_{p}}\left[4 \sqrt{2} c\left(\frac{e r_{e}}{l_{p}}\right)^{2}\left(\frac{r_{s}}{r}\right)^{2}\left(\frac{l_{p}}{r}\right)^{2}-2 \sqrt{2}(y+2 z)\left(\frac{e r_{e}}{l_{p}}\right)^{4}\left(\frac{r_{s}}{r}\right)^{3}\left(\frac{l_{p}}{r}\right)^{2}\right]
\end{aligned}
$$

and all other perturbations are vanishing or trivially related to the above.

Again, the result (5.14) only represents the dominant long distance corrections to the metric due to electrons; many subleading terms are neglected. For instance, for every diagram used in building the above, we could attach $n$ more external graviton lines to create a related diagram which is down by a factor of $\sim\left(r_{s} / r\right)^{n}$, relative to the original. These are all negligible for the interests of this paper, but are necessary for understanding the near horizon region, calculating how the fermion field affects the Hawking temperature, etc. Re-summing these subleading terms requires solving the fully non-linear EOM, while still working only to leading order in EFT coefficients (2.4). This is done in [87].

Massless loops dominate for $r \gtrsim r_{s}\left(\frac{e r_{e}}{l_{p}}\right)^{2}$ and writing their representation as a contribution to $\delta g_{\mu \nu}$ and $\delta F^{\mu \nu}$ is misleading due to the gauge loop subtleties covered in section 5.2.4. The precise one-loop potential generated by massless loops is calculated in appendix C, using the work of $[103,105,106]$, and is found to be of the expected, attractive $\delta V \sim \frac{r_{s}}{r}\left(\frac{l_{p}}{r}\right)^{2}$ form. 


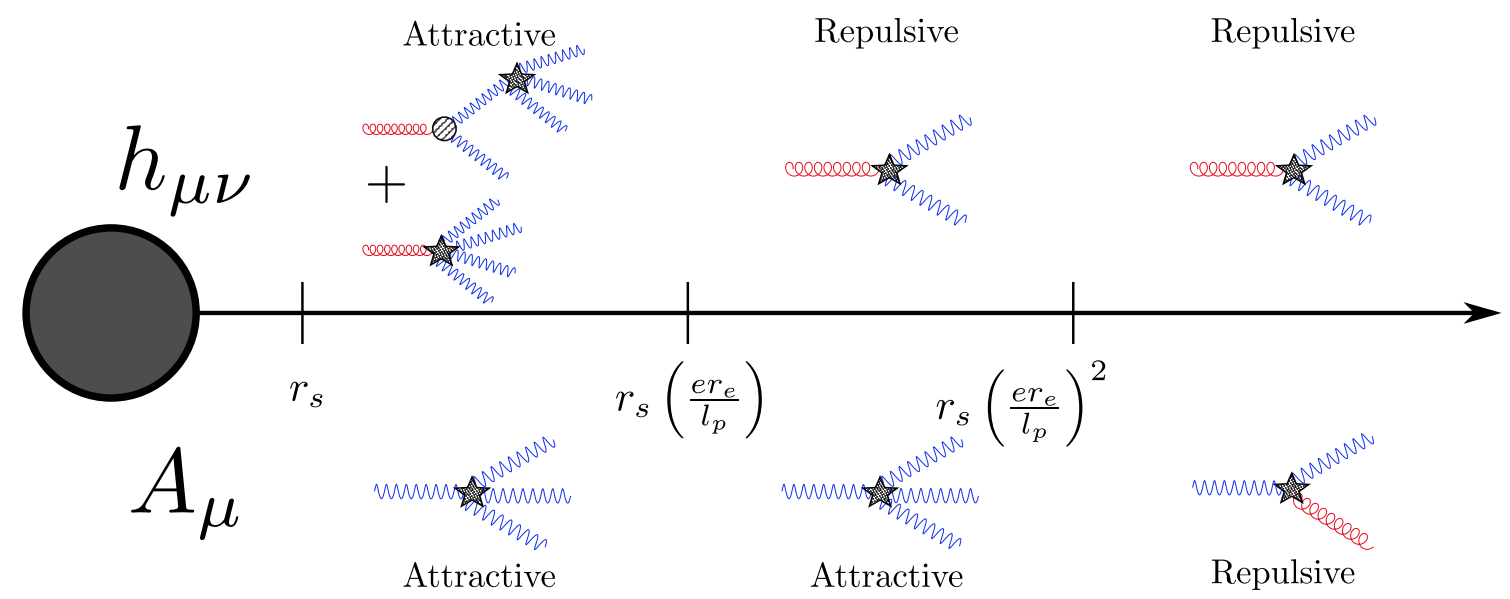

Figure 14. Dominant metric and vector potential corrections at different distances from the black hole when massless loops are neglected. By ignoring massless loops, qualitatively wrong dynamics are found.

Before we analyze the dynamics of the black hole ladder, we wish to quickly emphasize the importance of including gauge loops. Had they been neglected, we'd find qualitatively wrong physics. Including only the effects of electrons in the analysis, the sketch of the system would be changed from figure 13 to figure 14. The result is a hilltop potential which generates an attraction between extremal RN black holes separated by distances $r \lesssim r_{s}\left(\frac{e r_{e}}{l_{p}}\right)^{2}$ and a repulsion for those separated by $r \gtrsim r_{s}\left(\frac{e r_{e}}{l_{p}}\right)^{2}$. This is the behavior one would find by only perturbatively solving the equations of motion arising from (2.3).

\subsection{Tunnel dynamics and distance advance}

From the perturbative corrections (5.14), we can calculate the forces which act on the tunnel and ask whether it collapses before we are able to up a distance advance which parametrically violates the macroscopic superluminality bound, $\Delta X \gtrsim m_{e}^{-1}$. We find that no such violation is possible: our setup only approaches this bound from below and always remains a parametric distance away from saturation. Precisely, we find $\Delta X \lesssim e \times m_{e}^{-1}$. We ignore Hawking radiation and assume that the black holes retain a fixed charge-to-mass ratio throughout the process.

\subsubsection{Tunnel dynamics}

Consider the dynamics of a single pair of black holes. The entire tunnel would coalesce at least as quickly as this pair would, hence as a conservative estimate we need only look at the dynamics of this single pair. A particle of charge $q$ and mass $m$ traveling in some charged spacetime obeys the geodesic equation sourced by the Lorentz force law,

$$
\frac{\mathrm{d} x^{\nu}}{\mathrm{d} \tau} \nabla_{\nu} \frac{\mathrm{d} x^{\mu}}{\mathrm{d} \tau}=\frac{q}{e m} F_{\nu}^{\mu} \frac{\mathrm{d} x^{\nu}}{\mathrm{d} \tau} .
$$


We model the motion of the far separated black holes via the above relation. In the Newtonian limit, the spatial components of the above reduce to their familiar form,

$$
\frac{\mathrm{d}^{2} x^{i}}{\mathrm{~d} t^{2}}=\frac{1}{2} \partial_{i} h_{00}+\frac{q}{e m} F_{0}^{i}
$$

where $g_{\mu \nu}=\eta_{\mu \nu}+h_{\mu \nu}$.

We model the two black holes by two coupled Newtonian equations, each of the form (5.16). The two body problem can be reduced to an effective one-body problem for the separation between the black holes, in the usual way. Letting $r=\left|\vec{x}_{1}-\vec{x}_{2}\right|$ be the separation between the pair, the relation for the BH's becomes,

$$
\frac{\mathrm{d}^{2} r}{\mathrm{~d} t^{2}}=\partial_{i} h_{00}+\frac{\sqrt{2}}{e M_{p}} F_{0}^{i} .
$$

Since all background forces cancel (see appendix A), the leading force terms in (5.17) arise from the perturbative corrections found in the previous section (5.14) and those due to massless loops, as calculated in appendix C. The problem is therefore efficiently recast in terms of a conserved energy and effective (dimensionless) potential,

$$
E=\frac{1}{2} \dot{r}^{2}+V_{\text {eff }}(r)
$$

where $V_{\text {eff }}(r)$ descends from (5.14). We might also worry about subleading velocity dependent forces, but these can be neglected, as is justified in appendix A.

From the explicit form of the corrections discussed in the previous section, it can be determined that the effective potential $V_{\text {eff }}(r)$ has two distinct types of behavior, depending on the value of $r$ :

$$
V_{\mathrm{eff}}(r)= \begin{cases}-C_{1}\left(\frac{r_{s}}{r}\right)\left(\frac{l_{p}}{r}\right)^{2}, & r_{s}\left(\frac{e r_{e}}{l_{p}}\right)^{2} \lesssim r \\ -C_{2}\left(\frac{e r_{e}}{l_{p}}\right)^{4}\left(\frac{r_{s}}{r}\right)^{3}\left(\frac{l_{p}}{r}\right)^{2}, & r_{s} \lesssim r \lesssim r_{s}\left(\frac{e r_{e}}{l_{p}}\right)^{2} .\end{cases}
$$

There are only two distinct types of behavior above, despite the three different regimes presented in figure 13, because the electromagnetic corrections dominate the potential for all $r \lesssim r_{s}\left(e r_{e} / l_{p}\right)^{2}$. That is, at long distances, $r \gtrsim r_{s}\left(\frac{e r_{e}}{l_{p}}\right)^{2}$, massless loops generate the leading forces, while the $\sim F^{4} / m^{4}$ EFT operators generate the dominate forces at shorter scales. The $C_{i}$ 's are linear combinations of the $\mathcal{O}(1)$ coefficients which describe the effects of either electron (2.4) or gauge loops. Their precise values are not needed. Instead, it's only important we know they are positive so that the black hole pair is attracted at all distances.

It's easy to check that the dynamics stay non-relativistic throughout the collapse, justifying the use of (5.18). From (5.19), magnitude of potential is bounded by

$$
\left|V_{\max }\right| \lesssim\left(\frac{e r_{e}}{l_{p}}\right)^{4}\left(\frac{l_{p}}{r_{s}}\right)^{2}
$$


for $r \gtrsim r_{s}$. We previously found that the radius of the extremal black holes must satisfy $r_{s} \gg r_{e}\left(\frac{e r_{e}}{l_{p}}\right)$ to fall within the validity of the EFT description. Plugging this fact into (5.20) we find that the potential is bound by $\left|V_{\max }\right| \ll e^{2} \ll 1$, and hence the velocities which obey $v^{2} \sim V$ are also much smaller than unity, as we wanted to show. The pair's dynamics can then be tracked using Newtonian dynamics until the separation becomes $r \sim \mathcal{O}\left(r_{s}\right)$, at which point the perturbative treatment breaks down.

\subsubsection{Distance advance}

We now estimate the total distance advance acquired by $\gamma_{\text {QED }}$ as it passes through each region. Start by placing the pairs at rest with $r \rightarrow \infty$ and track the net distance advance gained by the photon.

If the black holes were all Schwarzschild, the velocity of the maximally superluminal photon would be similar to what we found in the Drummond-Hathrell section, schematically:

$$
c_{s} \approx 1+C_{4} \frac{e^{2} R_{\mu \nu \rho \sigma}}{m_{e}^{2}} .
$$

In (5.21), $C_{4}$ is a positive, $\mathcal{O}(1)$ number directly proportional to the EFT coefficient $c$ which also takes into account the geometry of the tunnel and $R_{\mu \nu \rho \sigma}$ represents the typical curvature felt by the photon when placed between a black hole pair.

The expression (5.21) only comes from considering the $\sim R F F / m_{e}^{2}$ terms in the EFT. When there are non-trivial electromagnetic sources, as in the present case, the $\sim F^{4} / m_{e}^{4}$ terms can also affect propagation, generically $[15,107]$. These operators decrease $c_{s}$ and generate physically relevant effects for pulsar physics ${ }^{21}$ of $\mathcal{O}(10 \%)$, see section 4 of the review [108] and references therein. However, in our current, highly symmetric scenario where the photon is sent directly between the black hole pair, the effects from each $\sim$ $F^{2 n} / m_{e}^{4 n-4}$ operator vanishes due to symmetry, as shown in appendix B. In many ways, the scenario we are considering is ideal for enhancing the superluminality, since these operators serve only to decrease $c_{s}$ in more generic setups.

In (5.8), the leading contribution to $R_{\mu \nu \rho \sigma}$ is of the form $R_{\mu \nu \rho \sigma} \sim \frac{r_{s}}{D^{3}}$ where $D$ is the distance between the photon and the nearest black hole pair. Therefore, $\delta c_{s}$ is wellapproximated by

$$
\delta c_{s} \approx C_{4} e^{2} \frac{r_{e}^{2} r_{s}}{r^{3}},
$$

with $r$ the black hole separation appearing in (5.19). Replacing $D$ by $r$ is a conservative estimate which approximates the setup by assuming that there's always a black hole directly on either side of the photon. For an appropriate choice of $C_{4} \sim \mathcal{O}(1)$, which accounts for both the EFT coefficient $c$ and the geometry of the tunnel, (5.22) serves as an upper bound on the velocity boost gained by the photon.

We now calculate the distance advanced gained by the maximally superluminal QED photon, relative to a minimally coupled photon, as it passes through the two regions described by (5.19):

\footnotetext{
${ }^{21}$ We thank Sam Gralla for bringing this fact to our attention.
} 
- In the outer region, $r \gtrsim r_{s}\left(\frac{e r_{e}}{l_{p}}\right)^{2}$, the distance advance gained is:

$$
\begin{aligned}
\Delta X & \approx \int_{t_{i}}^{t_{f}} \mathrm{~d} t \delta c_{s} \approx \int_{r_{s}\left(\frac{e r_{e}}{l_{p}}\right)^{2}}^{\infty} \mathrm{d} r \frac{\mathrm{d} t}{\mathrm{~d} r} \delta c_{s} \approx \int_{r_{s}\left(\frac{e r_{e}}{l_{p}}\right)^{2}}^{\infty} \mathrm{d} r \frac{\delta c_{s}}{\sqrt{-V_{\text {eff }}}} \\
& \approx \int_{r_{s}\left(\frac{e r_{e}}{l_{p}}\right)^{2}}^{\infty} \mathrm{d} r \frac{C_{4} e^{2} \frac{r_{e}^{2} r_{s}}{r^{3}}}{\sqrt{C_{1}\left(\frac{r_{s}}{r}\right)\left(\frac{l_{p}}{r}\right)^{2}}} \approx \frac{C_{4}}{\sqrt{C_{1}}} e m_{e}^{-1} .
\end{aligned}
$$

Here, and below, we drop $\mathcal{O}(1)$ numerical prefactors. The distance advance acquired is parametrically smaller than the cutoff of the EFT by a factor of the gauge coupling, $\Delta X \sim e \times m_{e}^{-1}$.

- The calculation is similar for the inner region, $r_{s} \lesssim r \lesssim r_{s}\left(\frac{e r_{e}}{l_{p}}\right)^{2}$ :

$$
\Delta X \approx \int_{r_{s}}^{r_{s}\left(\frac{e r_{e}}{l_{p}}\right)^{2}} \mathrm{~d} r \frac{C_{4} e^{2} \frac{r_{e}^{2} r_{s}}{r^{3}}}{\sqrt{C_{2}\left(\frac{e r_{e}}{l_{p}}\right)^{4}\left(\frac{r_{s}}{r}\right)^{3}\left(\frac{l_{p}}{r}\right)^{2}}} \approx \frac{C_{4}}{\sqrt{C_{2}}} e m_{e}^{-1} .
$$

Again, the distance advance is again parametrically smaller than the cutoff, $\Delta X \sim$ $e \times m_{e}^{-1}$.

Therefore, the total distance advance is parametrically smaller than the cutoff of the EFT.

The QED EFT appears to conspire in such a way that macroscopic superluminality is avoided. For instance, the transition between the two force laws behaviors occurs at exactly the scale it must to keep the distance advance parametrically suppressed. Had massless loops dominated down to, say, a distance scale $\sim r_{s}\left(\frac{e r_{e}}{l_{p}}\right)$ instead of $\sim r_{s}\left(\frac{e r_{e}}{l_{p}}\right)^{2}$, the distance advance gained by the photon in the outer region would have been $\Delta X \sim$ $e\left(\frac{e r_{e}}{l_{p}}\right)^{1 / 2} m_{e}^{-1}$ which can be consistently taken much larger than $m_{e}^{-1}$. For example, $e\left(\frac{e r_{e}}{l_{p}}\right)^{1 / 2} \sim 10^{12}$ in the Standard Model.

\subsubsection{Variations}

The above analysis can be refined and extended to variations of this scenario, but the conclusion remains the same: at worst, $\Delta X \approx e \times m_{e}^{-1}$.

Some examples:

- A simple extension is to start with the two sides of the black hole close together and give both sides an initial outwards velocity. However, this would extend the lifetime of the ladder (and hence, the overall distance advance) by a factor of two, at most.

- Instead of starting with the ladders infinitely far from each other, one could instead begin at finite separation. Though this shortens the overall lifetime of the latter, it also leads to a increase in the amount of time the ladder spends at relatively close separation where the superluminal effect is largest. However results are again the same to within a factor of $\mathcal{O}(2)$. 
- One could attempt to prolong the ladder lifetime by setting the sides of the ladder in orbit around each other. Though calculations are quite difficult in this setup, we do not expect such a configuration to perform any better than the non-rotating ladder for the simple reason that at some points in the cycle the configuration causes $c_{s}>1$ but at others $c_{s}<1$ and all effects should get washed out.

- Instead of considering just two columns of black holes, one could surround the photon's path by symmetric configurations with even more lines, thereby increasing the gravitational field of the system and potentially the size of $\delta c_{s}$ (5.21). For example, three or four lines of Schwarzschild black holes could be used to build setups which are triangular or square in cross section, respectively. However, in both scenarios the Drummond-Hathrell effect cancels completely for photons with arbitrary polarization, sent down the path of symmetry: $c_{s}=1$, at leading order. This pattern continues when even more lines are added and is another reason why the ladder seems to be the ideal setup for studying the effect.

An interesting possibility which deserves more detailed comment arises by overcharging the black holes. That is, in pure Einstein-Maxwell the black hole charge is bounded so that the inequality $r_{q} \leq r_{s}$ is satisfied. Otherwise, there's a naked singularity. However, in full QED where there are also fermionic fields, the bound is altered so that the black hole can carry slightly more charge ${ }^{22}[87,109]$ (see [110], also):

$$
r_{q} \lesssim r_{s}+\left(\frac{e r_{e}}{l_{p}}\right)^{4} \frac{l_{p}^{2}}{225 \pi^{2} r_{s}}-\left(\frac{e r_{e}}{l_{p}}\right)^{2} \frac{3 l_{p}^{2}}{225 \pi^{2} r_{s}}
$$

This is expected to be a generic property of theories which obey the WGC [71, 111]: black holes should allow for a maximum charge to mass ratio, $\max \left(r_{q} / r_{s}\right)$, which is slightly larger than unity and, further, smaller black holes should be able to carry proportionally more charge, $\frac{\mathrm{d}}{\mathrm{d} r_{s}} \max \left(r_{q} / r_{s}\right)<0$. Such properties prevent the existence of unnatural, exactly stable remnants whose stability doesn't follow from any symmetry principle [111].

By overcharging the black holes we can set up a hilltop type potential for the black holes which is attractive at short distances and then repulsive at large separation where the small $\sim 1 / r^{2}$ force due to overcharging begins to dominate. However, an analysis entirely analogous to that of the previous section demonstrates that we cannot use this effect to our advantage. The ladder either collapses too quickly, as before, or gets blown apart too fast, depending on the initial setup.

For instance, assuming $e r_{e} / l_{p} \gg 1$, extremal black holes in QED obey

$$
r_{q} \approx r_{s}\left(1+\frac{1}{225 \pi^{2}}\left(\frac{e r_{e}}{l_{p}}\right)^{4}\left(\frac{l_{p}}{r_{s}}\right)^{2}\right) .
$$

\footnotetext{
${ }^{22}$ This expression assumes the WGC, $e r_{e} / l_{p} \gg 1$. If the WGC is violated, then the $\sim R^{2}$ terms we've neglected in the action instead provide the leading corrections to this bound [87].
} 
The repulsive potential generated from overcharging dominates at all distances and is given by

$$
V \sim \frac{r_{s}}{r}\left(\frac{e r_{e}}{l_{p}}\right)^{4}\left(\frac{l_{p}}{r_{s}}\right)^{2}
$$

Releasing the ladder from an initial separation of $\mathcal{O}\left(r_{s}\right)$, it's found that we generate an asymptotic distance advance $\Delta X \sim l_{p} \ll m_{e}^{-1}$. There is no parametric win in any of these scenarios.

\subsubsection{Weak gravity conjecture}

One might wonder whether WGC-violating theories can achieve $\Delta d>m_{e}^{-1}$. It appears not to be the case. Assuming that $e r_{e} / l_{p} \ll 1$ and that the extremality bound for black holes still allows for $r_{q}=r_{s}$ (only true for certain coefficients on the $\sim R^{2}$ terms in the action), then graviton/photon loops generate the dominant large-distance corrections to the force law, $V \sim \frac{r_{s}}{r} \frac{l_{p}^{2}}{r^{2}}$. Releasing the black holes from infinity, the distance advance is

$$
\Delta d \sim \int_{r_{s}}^{\infty} \mathrm{d} r \frac{\delta c_{s}}{-\sqrt{V}} \sim \int_{r_{s}}^{\infty} \mathrm{d} r \frac{\frac{e^{2}}{m_{e}^{2}} \frac{r_{s}}{r^{3}}}{\sqrt{\frac{r_{s}}{r}} \frac{l_{p}^{2}}{r^{2}}} \sim\left(\frac{e r_{e}}{l_{p}}\right)^{2} l_{p} \ll l_{p} \ll r_{e} .
$$

While the force law is depressed in certain regions relative to that in WGC-obeying theories, the superluminal boost $\delta c_{s} \sim\left(\frac{e r_{e}}{l_{p}}\right)^{2}\left(\frac{l_{p}^{2} r_{s}}{r^{3}}\right)$ is also diminished. The WGC violating scenario is actually better behaved.

\subsubsection{Polarization rotation}

A final effect which fights against the generation of macroscopic distance advances in generic setup is the fact that in full QED photon polarizations rotate due to the different velocities for different polarization states in anisotropic backgrounds. While there may be some discrete polarization eigen-directions which travel with fixed polarization, a photon initially placed in a generic state will rotate into other ones as it propagates. This has been known for the case of electromagnetic backgrounds for some time [107], but is also true in gravitational backgrounds. The rotation tends to wash out any superluminal effects.

We now sketch how the effect arises, exploring it in more detail in [87]. It is found by pushing the geometric optics analysis to the next order, $\mathcal{O}\left(\epsilon^{-1}\right)$. The $\mathcal{O}\left(\epsilon^{-2}\right)$ geometric optics relation determined the dynamics of the wave vector through modifications of the geodesic equation. In pure Einstein-Maxwell, we'd have found $k^{\nu} \nabla_{\nu} k^{\mu}=0$, but when electrons are included the relation is changed to $k^{\nu} \nabla_{\nu} k^{\mu}=\mathcal{F}^{\mu}$ for some source term (see Footnote 17). This was rephrased as a true geodesic equation along an effective metric in Footnote 16. Similarly, in the absence of electrons, the $\mathcal{O}\left(\epsilon^{-1}\right)$ relation would read $k^{\nu} \nabla_{\nu} f_{\mu}=0$, where $f_{\mu}$ is the unit vector proportional to the polarization $a_{\mu}$, meaning that polarization is covariantly constant along the photon's trajectory. When electrons are included in the theory, we instead find

$$
k^{\nu} \nabla_{\nu} f_{\mu}=\Pi_{\mu}{ }^{\nu} \mathcal{S}_{\nu}
$$




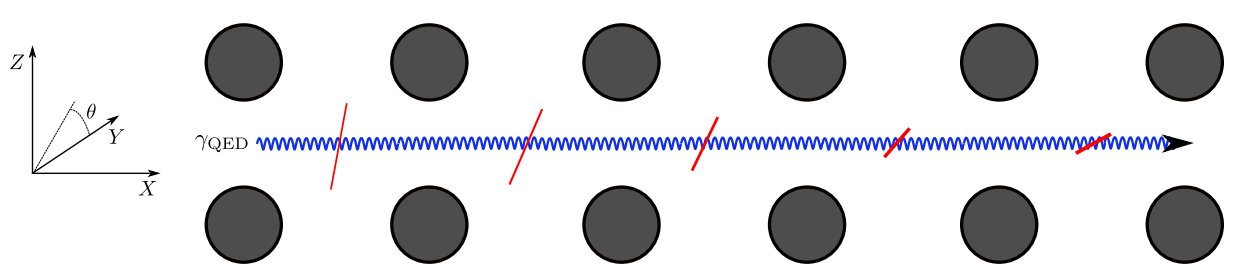

Figure 15. An exaggerated cartoon of polarization rotation for a photon traveling between a black hole ladder. The polarization vector is indicated by the red lines with the segments drawn shorter, thicker and more highly angled as the vectors point further and further into the page. The polarization angle starts near $\theta=\pi / 2$, corresponding a superluminal photon. This polarization is unstable and slowly rotates back down towards $\theta=0$.

In (5.29) $\Pi_{\mu}{ }^{\nu}=\delta_{\mu}^{\nu}-f_{\nu} f^{\mu}$ is the projection tensor constructed from $f_{\mu}$ and $\mathcal{S}_{\nu}$ is a source term depending on background curvatures, field strengths and properties of the wave whose form is given in [87].

Outside of a single black hole, only radially polarized photons travel superluminally. We find that the effects represented in (5.29) make this polarization unstable, while the azimuthal, subluminal polarization state is stable. That is, a photon which is initially polarized in a nearly (but not exactly) radial direction far from the black hole will have its polarization vector rotated further and further into the azimuthal direction as it nears the black hole. In contrast, a nearly azimuthally polarized photon becomes even further azimuthally polarized as it approaches the black hole. The gravitational field breaks the symmetry between the two polarization states and induces a preferred direction for the vector. To our knowledge, the effect of the Dirac field on the polarization of a propagating photon due to gravitational fields has not been studied before.

The rotation is minuscule, but it could certainly become relevant in thought experiments like the black hole ladder of figure 9. Here, if the QED photon started with a nearly maximally superluminal polarization vector, $\theta_{0}=\pi / 2-\delta$ with $\delta>0$, then as it passes through the first black hole pair, the angle would be slightly rotated down to some $\theta_{1}<\theta_{0}$. The difference between the two angles would be tiny, but it sets the initial condition for $\theta$ as $\gamma_{\text {QED }}$ passes through the next pair, after which the polarization angle will be again rotated down to some $\theta_{2}<\theta_{1}$. This process continues and $\gamma_{\text {QED }}$ gets smaller and smaller superluminal kicks as the travel continues, with the velocity turning subluminal at some point. This is sketched in figure 15 .

For emphasis, this rotation is not being generated by the existence of free charged particles. It is an effect which persists in vacuum and is the result of having "virtual" electrons and positrons which exist because of the Dirac field.

\section{Galileon superluminality}

We now turn to the superluminality which arises in the simplest galileon model (1.5). First the background is derived, then the geometric optics analysis is carried out and, finally, we race a galileon perturbation against a photon, showing that the superluminality is of a qualitatively different magnitude. Gravity is ignored in this section. 


\subsection{Background solution}

Consider the cubic galileon coupled to a point mass (1.5),

$$
\mathcal{L}=-\frac{1}{2}(\partial \pi)^{2}-\frac{1}{\Lambda^{3}}(\square \pi)(\partial \pi)^{2}+\frac{1}{M_{p}} \pi T_{\mu}^{\mu}, \quad T_{\mu}{ }^{\mu}=-M \delta^{3}(r) .
$$

The galileon equations of motion are particularly simple as they admit a first integral [33]:

$$
0=\frac{\delta \mathcal{L}}{\delta \pi}=\frac{1}{r^{2}} \frac{\mathrm{d}}{\mathrm{d} r}\left(r^{2} \pi^{\prime}\right)+\frac{4}{\Lambda^{3} r^{2}} \frac{\mathrm{d}}{\mathrm{d} r}\left(r \pi^{\prime}\right)+T^{\mu}{ }_{\mu} / M_{p}
$$

where $\pi^{\prime}=\frac{\mathrm{d} \pi}{\mathrm{d} r}$. Solving, one finds two distinct behaviors for $\pi(r)$, depending on whether $r$ is much larger or smaller than the Vainshtein radius of the source $r_{V}=\Lambda^{-1}\left(M / M_{p}\right)^{1 / 3}$ :

$$
\pi(r) \approx \begin{cases}-\frac{1}{l_{p}} \frac{r_{s}}{r} & r \gg r_{V} \\ \frac{1}{\sqrt{4 \pi}} \frac{1}{l_{p}} \frac{r_{s}}{r}\left(\frac{r}{r_{V}}\right)^{3 / 2} & r \ll r_{V}\end{cases}
$$

\subsection{Geometric optics analysis}

Now we apply geometric optics to the propagation of perturbations about the background solution. We let $\pi=\bar{\pi}+\delta \pi$ with $\delta \pi(x)=(a+\epsilon b+\ldots) \exp i \vartheta(x) / \epsilon$ and expand the equation of motion to first order in $\delta \pi$. The background equation of motion is

$$
\square \pi-\frac{2}{\Lambda^{3}}\left(\partial^{\mu} \partial^{\nu} \pi \partial_{\mu} \partial_{\nu} \pi-(\square \pi)^{2}\right)=-\frac{T_{\mu}^{\mu}}{M_{p}},
$$

and the $\mathcal{O}(\delta \pi)$ piece is

$$
\square \delta \pi=\frac{4}{\Lambda^{3}}\left(\partial^{\mu} \partial^{\nu} \bar{\pi} \partial_{\mu} \partial_{\nu} \delta \pi-\square \bar{\pi} \square \delta \pi\right)
$$

Therefore, the leading term in the geometric optics EOM is

$$
\tilde{g}^{\mu \nu} k_{\mu} k_{\nu}=0, \quad \tilde{g}^{\mu \nu}=\eta^{\mu \nu}+\frac{4}{\Lambda^{3}} \square \bar{\pi} \eta^{\mu \nu}-\frac{4}{\Lambda^{3}} \partial^{\mu} \partial^{\nu} \bar{\pi} .
$$

Following the same steps as the previous section, we can study the galileon trajectory by finding the geodesics of the optical metric $\tilde{g}_{\mu \nu}$. Working at distances $r \gtrsim r_{V}$ and parameterizing the geodesic such that $X=X_{0} \gtrsim r_{V}$ at $\lambda=0$, we find

$$
x^{\mu}(\lambda) \approx\left(\lambda, X_{0}+\lambda+\frac{r_{V}^{3} \lambda\left(\lambda+2 X_{0}\right)}{2 \pi X_{0}^{2}\left(\lambda+X_{0}\right)^{2}}, 0,0\right) .
$$

\subsection{Racing $\delta \pi$ against a photon}

We can now compare the galileon geodesic to that of a test photon which also travels from $X_{0}$ to infinity. The photon's geodesics are manifestly unaffected by the galileon field, since 
the $\sim \pi T^{\mu}{ }_{\mu}$ coupling vanishes for the Maxwell term. ${ }^{23}$ Racing from $X_{0} \sim \mathcal{O}\left(r_{V}\right)$ out to infinity, it's found from (6.7) that the galileon perturbation beats the photon by a distance

$$
\Delta X=\lim _{\lambda \rightarrow \infty} x^{1}(\lambda)=\frac{r_{V}}{2 \pi}\left(\frac{r_{V}}{X_{0}}\right)^{2},
$$

which is $\mathcal{O}\left(r_{V}\right) \gg \Lambda^{-1}$, as previously estimated (1.7) (see [61], also).

\section{Conclusions}

We have studied the Drummond-Hathrell superluminality present in the low energy effective field theory obtained by integrating the electron out of QED coupled to gravity. This effective field theory has a cutoff at a distance scale corresponding to the Compton wavelength of the electron, $m_{e}^{-1}$. If the full QED theory does not allow for superluminality, and no strong coupling effects, new particles or other non-perturbative effects come in before $m_{e}^{-1}$, then any distance advance $\Delta X$ generated by a superluminal photon along any trajectory in any background of the effective theory must not be resolvable in the EFT, so we must have $\Delta X \lesssim m_{e}^{-1}$. We have tested this assertion by attempting to contrive various backgrounds to amplify the superluminal effects, and indeed in all the cases we try the distance advance is smaller than $m_{e}^{-1}$.

The main scenario we consider is building distance advances via a ladder of approximately extremal, Reissner-Nordstrom black holes. In order to account for all relevant effects, we not only needed to find the perturbative corrections to the RN solution to the higher-derivative, electron-induced operators in the QED EFT, but also needed to consider the subtle effects of graviton and photon loops. In the end, the distance advance we were capable of generating was parametrically bounded by $\Delta X \lesssim e \times m_{e}^{-1}$ with $e$ the QED gauge coupling.

We then compared this to the analogous story for the galileons. Unlike the QED case, we do not know of a local weakly coupled UV completion for the galileon (and there exist argument against any such completion [50]). All we have is the low energy effective theory, which comes with a strong coupling distance scale $\Lambda^{-1}$. The superluminal distance advances in the galileon case can easily be made much larger than $\Lambda^{-1}$, and are typically as large as the Vainshtein radius, $r_{V}$, associated with the background. It should, however, be noted that it proves very difficult to generate distance advances parametrically larger than $r_{V}$.

If the underlying UV theory for the galileons is indeed subluminal, then the UV completion must proceed in a qualitatively different way than it does in the QED case. It cannot be simply be a new weakly coupled particle coming in at the scale $\Lambda$. In order to cure the superluminality, there must instead be strong coupling effects or strong quantum effects coming in at the background-dependent scale $r_{V}$.

\footnotetext{
${ }^{23}$ Other massless species also end up traveling along their Minkowski geodesics because the $\sim \pi T^{\mu}{ }_{\mu}$ coupling results in an effective optical metric $\tilde{g}_{\mu \nu}$ which is conformally flat, but the argument is especially simple for photons.
} 
This kind of situation is also thought to occur in GR. In GR, the Schwarzschild radius is the scale at which non-linearities become important, and plays the role of the Vainshtein radius of the galileon theories. The black hole information paradox, along with the assumptions of unitarity and the equivalence principle, tell us that strict locality must break down at the scale of the horizon, so that the information may escape from the black hole. Quantum gravity effects, which are completely invisible from the point of view of the low energy local effective field theory, must come in at the scale of the horizon and mediate these non-localities [112].

A similar picture could hold true for the galileons, consistent with the findings of [67], and with the classicalization ideas of [66]. If so, then the true physics of galileon-like theories is highly non-perturbative in the quantum sense (not just classically non-linear), at all scales within the Vainshtein regime, which includes essentially all scales of phenomenological interest. This of course does not mean it's ruled out, only that it is difficult to calculate anything with it.

Alternatively, one can impose boundary conditions on the theory so that only backgrounds which do not possess large scale superluminality are available, e.g. [58]. In this way, the above conclusions can be avoided, without sacrificing UV subluminality. However, we should also keep in mind that it is also logically possible to simply withdraw the demand that the UV theory be subluminal, in which case the above does not have to apply, and the superluminality of the low energy galileons is physical.

\section{Acknowledgments}

It is our pleasure to thank Daniel Baumann, David Chernoff, Claudia de Rham, Ian Drummond, Siavash Golkar, Austin Joyce, Justin Khoury, Guilherme Pimentel, Rachel Rosen and Mark Trodden for discussions and comments on the draft. GG gratefully acknowledges support from a Starting Grant of the European Research Council (ERC StG grant 279617). We thank the participants and organizers of the Superluminality in Effective Field Theories for Cosmology workshop at Perimeter Institute where this work originated. This work was also performed in part at the Aspen Center for Physics, which is supported by National Science Foundation grant PHY-1066293 (GG).

\section{A Velocity dependent forces}

In this appendix, we estimate the sizes of various velocity dependent effects and verify that they are negligible.

First, we explore the stability of the multi-extremal RN solution (5.4) by adding a new extremal black hole to the spacetime and calculating the forces acting upon it. If the new black hole is placed at rest relative to the other black holes, then the system is perfectly stable, but if it's in motion, forces are generated.

Placing the new black hole far from the others, we can analyze its motion with the modified geodesic equation appropriate for a particle with charge $Q$ and mass $M$ :

$$
\frac{\mathrm{d} x^{\nu}}{\mathrm{d} \tau} \nabla_{\nu} \frac{\mathrm{d} x^{\mu}}{\mathrm{d} \tau}=\frac{Q}{e M} F_{\nu}^{\mu} \frac{\mathrm{d} x^{\nu}}{\mathrm{d} \tau} .
$$


The spacetime has a timelike Killing vector $K=\partial_{t}$, implying that the following is conserved:

$$
C \equiv-K_{\mu}\left(\frac{\mathrm{d} x^{\mu}}{\mathrm{d} \tau}+\frac{Q}{e M} A^{\mu}\right)
$$

Evaluating the spatial components of the modified geodesic equation (A.1) at $\frac{\mathrm{d} X^{i}}{\mathrm{~d} \tau}=0$ and using (A.2) and $-1=g_{\mu \nu} \frac{\mathrm{d} x^{\mu}}{\mathrm{d} \tau} \frac{\mathrm{d} x^{\nu}}{\mathrm{d} \tau}$ yields the acceleration for the initially stationary probe particle:

$$
\frac{\mathrm{d}^{2} X^{i}}{\mathrm{~d} \tau^{2}}=-\frac{1}{2}\left(1-\frac{\sqrt{2} M_{p} Q}{M}\right) \partial_{i}\left(U^{-2}\right) .
$$

This is vanishing only if the new $\mathrm{BH}$ is extremal and carries the same sign charge as the original BHs: $Q=\frac{M}{\sqrt{2} M_{p}}$.

Next, we can calculate the forces which act on the new, extremal BH if it were moving with some velocity $\frac{\mathrm{d} X^{i}}{\mathrm{~d} \tau} \neq 0$. If the instantaneous velocity is $v^{2}=\delta_{i j} \frac{\mathrm{d} X^{i}}{\mathrm{~d} \tau} \frac{\mathrm{d} X^{j}}{\mathrm{~d} \tau}$, the spatial geodesic equations read (to first order in $v^{2}$ ):

$$
\frac{\mathrm{d}^{2} X^{i}}{\mathrm{~d} \tau^{2}}=\frac{v^{2}}{2} U^{-2} \partial_{i} U
$$

Recalling that $U=1+\sum_{i} \frac{r_{s}}{2\left|\vec{X}-\vec{X}_{i}\right|}$ (5.4), it's found that (A.4) correspond to a small attraction between the probe particle and the original BHs. The origin of this attraction is clear: when the new $\mathrm{BH}$ is stationary, the gravitational attraction generated by its energy is perfectly tuned to cancel off the electromagnetic repulsion due to the BH's charge. Therefore, when in motion, the BH carries some additional kinetic energy, leading to a slightly increased gravitational (and therefore overall) attraction.

For our interests, (A.4) is important because it justifies the neglect of such velocitydependent forces in our analysis of the black hole ladder in section 5. At any given moment, the velocity of a black hole in the ladder is of order the potential generated from electroninduced EFT corrections to the gravitational and electromagnetic background, $v^{2} \sim V_{\mathrm{EFT}}$, schematically. The velocity-dependent force is thus of size $F_{\mathrm{v} . \mathrm{d} .} \sim v^{2} \frac{r_{s}}{r^{2}} \sim \frac{r_{s}}{r^{2}} V_{\mathrm{EFT}}$, while the EFT forces are of size $F_{\mathrm{EFT}} \sim \partial_{r} V_{\mathrm{EFT}} \sim \frac{1}{r} V_{\mathrm{EFT}}$. The velocity dependent force is therefore suppressed relative to the EFT forces by a factor of $r_{s} / r$ and are thus dominated in our regime of interest.

Next, we can also consider radiation reaction forces. We will show they they are also negligible, meaning that the black holes don't radiate significantly as they accelerate towards one another. The Abraham-Lorentz law corresponds to a force $F_{\mathrm{AL}} \sim Q^{2} \dot{a}$ where $a$ is the acceleration of the charged object. As $M a \sim \frac{1}{r} \sqrt{V_{\mathrm{EFT}}}$, we have

$$
\dot{a} \sim \frac{1}{M} \frac{\mathrm{d}}{\mathrm{d} t}\left(\frac{1}{r} \sqrt{V_{\mathrm{EFT}}}\right) \sim \frac{v}{M r^{2}} \sqrt{V_{\mathrm{EFT}}} \sim \frac{1}{M r^{2}} V_{\mathrm{EFT}},
$$

and hence the size of this effect is

$$
F_{\mathrm{AL}} \sim \frac{Q^{2}}{M r} \frac{1}{r} V_{\mathrm{EFT}} \sim \frac{Q^{2}}{M r} F_{\mathrm{EFT}}
$$

For extremal objects, $\frac{Q^{2}}{M r} \sim \frac{r_{s}}{r}$ and hence the radiation reaction force is, again, smaller than the leading forces by a factor of $r_{s} / r \ll 1$ and is negligible. 


\section{B Effects of $F^{2 n} / m_{e}^{4 n-4}$ operators on $c_{s}$}

In this appendix we demonstrate that none of the $F^{2 n} / m_{e}^{4 n-4}$ operators in the EFT affect $c_{s}$ in our very specific setup.

The EOM for photon fluctuations is of the form $\nabla_{\nu} \delta F^{\nu}{ }_{\mu}=\nabla_{\nu} \frac{\delta \mathcal{L}_{\text {eff }}}{\delta F_{\nu} \mu}$ and if we work in Lorenz gauge for the fluctuation (implying $a_{\mu} k^{\mu}=0$ (3.6)), then the geometric optics dispersion relation follows from $k^{2} \propto a^{\mu} \nabla_{\nu} \delta F^{\nu}{ }_{\mu}=a^{\mu} \nabla_{\nu} \frac{\delta \mathcal{L}_{\text {eff }}}{\delta F_{\nu}}$, where only the $\mathcal{O}\left(\epsilon^{-2}\right)$ $\operatorname{parts}^{24}$ of $a^{\mu} \nabla_{\nu} \delta F^{\nu}$ and $a^{\mu} \nabla_{\nu} \frac{\delta \mathcal{L}_{\text {eff }}}{\delta F_{\nu}{ }^{\mu}}$ are kept.

Consider the terms in $\mathcal{L}_{\text {eff }}$ of the form $\sim F^{2 n} / m_{e}^{4 n-4}$. From the explicit expressions for the Euler-Heisenberg action [113], each term can be put in to the form $\sim\left(F_{\mu \nu} F^{\mu \nu}\right)^{i}\left(F_{\mu \nu} \tilde{F}^{\mu \nu}\right)^{j}$ where, by parity conservation, $j$ is even. Because $F \tilde{F} \sim E \cdot B$ and $B=0$ along the photon's path (by the symmetry of the problem), terms with $j \geq 4$ have no effect on the dispersion relation, as their contribution is proportional to a power of $F \tilde{F}$. The $j=2$ case needs to be treated separately since it can yield a nontrivial term:

$$
a_{\mu} \nabla_{\nu} \frac{\delta \mathcal{L}_{\mathrm{eff}}}{\delta F_{\nu} \mu} \sim(F F)^{i}\left(a_{\mu} k_{\nu} \tilde{F}^{\mu \nu}\right)^{2}
$$

where we've dropped other vanishing contributions $\propto F \tilde{F}$. On the line $Z=Y=0$, the only non-vanishing components of $F^{\mu \nu}$ is $F^{t X}=-F^{X t}$, and hence the only non-vanishing component of $\tilde{F}^{\mu \nu}$ is $\tilde{F}^{Y Z}=-\tilde{F}^{Z Y}$. Therefore, $a_{\mu} k_{\nu} \tilde{F}^{\mu \nu}$ vanishes for the trajectory we are interested in, since $k^{\mu} \sim(1,1,0,0)$, to the order needed for this calculation.

The remaining $\sim(F F)^{i}$ terms yield contributions of the form $a^{\mu} \nabla_{\nu} \frac{\delta \mathcal{L}_{\text {eff }}}{\delta F_{\nu} \mu} \sim$ $a_{\mu} \nabla_{\nu}\left[(F F)^{i-1} F^{\mu \nu}\right]$. These generate two different types of expressions which are either proportional to $a_{\mu} k_{\nu} a^{[\mu} k^{\nu]}$ or $a_{\mu} k_{\nu} F^{\mu \nu}$. The first expression either vanishes by the gauge condition or is $\propto k^{2}$ which represents a higher order effect (quadratic in EFT coefficients). The second combination, $a_{\mu} k_{\nu} F^{\mu \nu}$, vanishes along the photon's path, due to the fact that $a_{\mu}$ points in the $Y-Z$ plane, ${ }^{25}$ while only $F^{t X}=-F^{X t}$ is non-zero along the trajectory.

Therefore, none of the $\sim F^{2 n} / m_{e}^{4 n-4}$ operators affect $c_{s}$ for this very tuned scenario, as claimed.

\section{One-loop potential from massless fields}

In this appendix, we use the results of $[103,105,106]$ to calculate the one-loop correction to the potential due to massless graviton and photon loops.

First, $[105,106]$ calculated the one-loop, $\mathcal{O}\left(Q^{2} / M_{p}^{2}\right)$ contribution to the non-relativistic potential in scalar QED (where $Q$ is the charge of $\phi$ ) due to mixed photon-graviton scattering diagrams. For equal charges and masses, the result is a repulsive potential:

$$
\mathcal{V}_{\mathcal{O}\left(Q^{2} / M_{p}^{2}\right)}=\frac{5 e^{2} Q^{2}}{48 M_{p}^{2} \pi^{3} r^{3}},
$$

\footnotetext{
${ }^{24}$ See section 6.2 for the review of geometric optics and the definition of $\epsilon$.

${ }^{25}$ More precisely, to zeroth order in EFT coefficients, which is all we need for this calculation, the gauge condition only determines $a_{\mu}$ up to an equivalence class: $a_{\mu} \sim a_{\mu}+k_{\mu}$. All elements in the class lead to the same result for $a_{\mu} k_{\nu} F^{\mu \nu}$, due to the antisymmetry of $F^{\mu \nu}$, and it's possible to work with a representative element, $a_{\mu}$, which lies in the $Y-Z$ plane.
} 
where we took the equal mass, equal charge limit of the $\mathcal{O}(\hbar)$ part of eq. (41) in [106], or eq. (58) in [105], and translated conventions. We use the symbol $\mathcal{V}$ for the dimensionful potential to differentiate it from the dimensionless potential $V$ used in the body of the paper, as in (5.18) and following expressions.

Next, [103] found the one-loop correction to the non-relativistic potential between two masses. This was calculated in the context of pure GR where the following $\mathcal{O}\left(M^{2} / M_{p}^{4}\right)$ attractive correction between two equal-mass particles was found

$$
\mathcal{V}_{\mathcal{O}\left(m^{2} / M_{p}^{4}\right)}^{\mathrm{GR}}=-\frac{41 M^{2}}{640 M_{p}^{4} \pi^{3} r^{3}}
$$

in our conventions, from their eq. (44).

However, because (C.2) was obtained in GR, it's not immediately applicable to the scenario considered in this paper. We also need to include the $\mathcal{O}\left(M^{2} / M_{p}^{4}\right)$ contribution from photon loops. Fortunately, this is a simple fix: one only needs to change the vacuum polarization diagram so that both gravitons and photons (along with their associated ghosts) run the loop, figures 6(a) and 6(b) in [103].

Vacuum polarization diagrams involving massless loops generate non-analytic terms in the 1 PI effective action of the form ${ }^{26}$ (5.13)

$$
\Gamma\left[\bar{g}_{\mu \nu}\right] \supset \int \mathrm{d}^{4} x \sqrt{-\bar{g}}\left(c_{1} \bar{R} \log \left(-\square / \mu^{2}\right) \bar{R}+c_{2} \bar{R}^{\mu \nu} \log \left(-\square / \mu^{2}\right) \bar{R}_{\mu \nu}\right) .
$$

The contribution of these operators to the potential (C.2) is:

$$
\mathcal{V}_{R \log \square R}=\left(c_{1}+c_{2}\right) \frac{M^{2}}{M_{p}^{4} \pi r^{3}},
$$

and [103] used the 't Hooft-Veltman [88], pure GR result

$$
\left(\begin{array}{l}
c_{1}^{\mathrm{GR}} \\
c_{2}^{\mathrm{GR}}
\end{array}\right)=\frac{1}{(4 \pi)^{2}}\left(\begin{array}{c}
-\frac{1}{120} \\
-\frac{7}{20}
\end{array}\right)
$$

as calculated in Feynman gauge, which provided the following contribution to (C.2)

$$
\mathcal{V}_{R \log \square R}^{\mathrm{GR}}=\frac{-43}{1920} \frac{M^{2}}{M_{p}^{4} \pi^{3} r^{3}},
$$

see eq. (43) of [103].

When photons are included, they also contribute an amount $c_{i}^{\gamma}$ to the $c_{i}$ 's, with the total result $c_{i}=c_{i}^{\mathrm{GR}}+c_{i}^{\gamma}$. Calculating the necessary vacuum polarization diagrams, we find

$$
\left(\begin{array}{l}
c_{1}^{\gamma} \\
c_{2}^{\gamma}
\end{array}\right)=\frac{1}{(4 \pi)^{2}}\left(\begin{array}{c}
\frac{1}{30} \\
-\frac{1}{10}
\end{array}\right),
$$

\footnotetext{
${ }^{26}$ Strictly speaking, we'd need to also have a $\sim \bar{R}^{\mu \nu \rho \sigma} \log \left(-\square / \mu^{2}\right) \bar{R}_{\mu \nu \rho \sigma}$, but at $\mathcal{O}\left(h_{\mu \nu}^{2}\right)$, sufficient for the present calculation, the three $\mathcal{O}\left(R^{2}\right)$ operators are degenerate and the form (C.3) is adequate.
} 
(in agreement with [90]) which generates the extra, attractive potential

$$
\mathcal{V}_{R \log \square R}^{\gamma}=-\frac{131}{1920} \frac{M^{2}}{M_{p}^{4} \pi^{3} r^{3}} .
$$

Adding (C.1), (C.2) and (C.8) together and taking the extremal limit $e Q=\frac{M}{M_{p} \sqrt{2}}$, the total one-loop contribution to the potential due to graviton and photon loops is found to be

$$
\mathcal{V}_{\text {total }}^{\mathrm{GR}+\gamma}=-\frac{23}{1920} \frac{M^{2}}{M_{p}^{4} \pi^{3} r^{3}}
$$

which is attractive and of the claimed form (5.19) (when turned into a dimensionless potential via $V \sim \mathcal{V} / M)$.

Open Access. This article is distributed under the terms of the Creative Commons Attribution License (CC-BY 4.0), which permits any use, distribution and reproduction in any medium, provided the original author(s) and source are credited.

\section{References}

[1] E. Babichev, V. Mukhanov and A. Vikman, k-Essence, superluminal propagation, causality and emergent geometry, JHEP 02 (2008) 101 [arXiv:0708.0561] [INSPIRE].

[2] R. Geroch, Faster Than Light?, AMS/IP Stud. Adv. Math. 49 (2011) 59 [arXiv: 1005.1614] [INSPIRE].

[3] C. Burrage, C. de Rham, L. Heisenberg and A.J. Tolley, Chronology Protection in Galileon Models and Massive Gravity, JCAP 07 (2012) 004 [arXiv:1111.5549] [INSPIRE].

[4] G. Papallo and H.S. Reall, Graviton time delay and a speed limit for small black holes in Einstein-Gauss-Bonnet theory, JHEP 11 (2015) 109 [arXiv:1508.05303] [INSPIRE].

[5] J. Friedman et al., Cauchy problem in space-times with closed timelike curves, Phys. Rev. D 42 (1990) 1915 [INSPIRE].

[6] I.T. Drummond and S.J. Hathrell, QED Vacuum Polarization in a Background Gravitational Field and Its Effect on the Velocity of Photons, Phys. Rev. D 22 (1980) 343 [INSPIRE].

[7] S. Dubovsky, A. Nicolis, E. Trincherini and G. Villadoro, Microcausality in curved space-time, Phys. Rev. D 77 (2008) 084016 [arXiv:0709.1483] [InSPIRE].

[8] G.M. Shore, 'Faster than light' photons in gravitational fields: Causality, anomalies and horizons, Nucl. Phys. B 460 (1996) 379 [gr-qc/9504041] [INSPIRE].

[9] G.M. Shore, Quantum gravitational optics, Contemp. Phys. 44 (2003) 503 [gr-qc/0304059] [INSPIRE].

[10] G.M. Shore, Superluminality and UV completion, Nucl. Phys. B 778 (2007) 219 [hep-th/0701185] [INSPIRE].

[11] I.B. Khriplovich, Superluminal velocity of photons in a gravitational background, Phys. Lett. B 346 (1995) 251 [gr-qc/9411052] [INSPIRE]. 
[12] S. Mohanty and A.R. Prasanna, Photon propagation in Einstein and higher derivative gravity, Nucl. Phys. B 526 (1998) 501 [gr-qc/9804017] [INSPIRE].

[13] G. Preti, A note on the geometrical-optics solution to the Maxwell tensor wave equation in curved spacetime, Nucl. Phys. B 834 (2010) 390 [INSPIRE].

[14] R. Akhoury and A.D. Dolgov, On the Possibility of Super-luminal Propagation in a Gravitational Background, arXiv:1003.6110 [INSPIRE].

[15] R.D. Daniels and G.M. Shore, 'Faster than light' photons and charged black holes, Nucl. Phys. B 425 (1994) 634 [hep-th/9310114] [INSPIRE].

[16] R.D. Daniels and G.M. Shore, 'Faster than light' photons and rotating black holes, Phys. Lett. B 367 (1996) 75 [gr-qc/9508048] [INSPIRE].

[17] T.J. Hollowood and G.M. Shore, Causality and Micro-Causality in Curved Spacetime, Phys. Lett. B 655 (2007) 67 [arXiv:0707.2302] [INSPIRE].

[18] T.J. Hollowood and G.M. Shore, The refractive index of curved spacetime: The fate of causality in QED, Nucl. Phys. B 795 (2008) 138 [arXiv:0707.2303] [InSPIRE].

[19] T.J. Hollowood and G.M. Shore, The Causal Structure of QED in Curved Spacetime: Analyticity and the Refractive Index, JHEP 12 (2008) 091 [arXiv:0806.1019] [INSPIRE].

[20] T.J. Hollowood, G.M. Shore and R.J. Stanley, The Refractive Index of Curved Spacetime II: QED, Penrose Limits and Black Holes, JHEP 08 (2009) 089 [arXiv:0905.0771] [InSPIRE].

[21] T.J. Hollowood and G.M. Shore, The Effect of Gravitational Tidal Forces on Vacuum Polarization: How to Undress a Photon, Phys. Lett. B 691 (2010) 279 [arXiv:1006.0145] [INSPIRE].

[22] T.J. Hollowood and G.M. Shore, 'Superluminal' Photon Propagation in QED in Curved Spacetime is Dispersive and Causal, arXiv:1006.1238 [INSPIRE].

[23] T.J. Hollowood and G.M. Shore, The Effect of Gravitational Tidal Forces on Renormalized Quantum Fields, JHEP 02 (2012) 120 [arXiv:1111.3174] [INSPIRE].

[24] T.J. Hollowood and G.M. Shore, The Unbearable Beingness of Light, Dressing and Undressing Photons in Black Hole Spacetimes, Int. J. Mod. Phys. D 21 (2012) 1241003 [arXiv:1205.3291] [INSPIRE].

[25] T.J. Hollowood and G.M. Shore, Causality Violation, Gravitational Shockwaves and UV Completion, JHEP 03 (2016) 129 [arXiv:1512.04952] [INSPIRE].

[26] T.J. Hollowood and G.M. Shore, Causality, Renormalizability and Ultra-High Energy Gravitational Scattering, J. Phys. A 49 (2016) 215401 [arXiv: 1601.06989] [InSPIRE].

[27] J.Z. Simon, Higher Derivative Lagrangians, Nonlocality, Problems and Solutions, Phys. Rev. D 41 (1990) 3720 [INSPIRE].

[28] X. Jaen, J. Llosa and A. Molina, A reduction of order two for infinite order lagrangians, Phys. Rev. D 34 (1986) 2302 [InSPIRE].

[29] C.P. Burgess and M. Williams, Who You Gonna Call? Runaway Ghosts, Higher Derivatives and Time-Dependence in EFTs, JHEP 08 (2014) 074 [arXiv: 1404.2236] [INSPIRE].

[30] L. Brillouin, Wave Propagation and Group Velocity, Series in Pure \& Applied Physics), Elsevier (1960). 
[31] P. Milonni, Fast Light, Slow Light and Left-Handed Light, Series in Optics and Optoelectronics, CRC Press (2004).

[32] C. de Rham, Massive Gravity, Living Rev. Rel. 17 (2014) 7 [arXiv:1401.4173] [InSPIRE].

[33] A. Nicolis, R. Rattazzi and E. Trincherini, The Galileon as a local modification of gravity, Phys. Rev. D 79 (2009) 064036 [arXiv:0811.2197] [INSPIRE].

[34] G.R. Dvali, G. Gabadadze and M. Porrati, $4-D$ gravity on a brane in $5-D$ Minkowski space, Phys. Lett. B 485 (2000) 208 [hep-th/0005016] [INSPIRE].

[35] M.A. Luty, M. Porrati and R. Rattazzi, Strong interactions and stability in the DGP model, JHEP 09 (2003) 029 [hep-th/0303116] [INSPIRE].

[36] C. de Rham, G. Gabadadze and A.J. Tolley, Resummation of Massive Gravity, Phys. Rev. Lett. 106 (2011) 231101 [arXiv: 1011.1232] [INSPIRE].

[37] K. Hinterbichler, Theoretical Aspects of Massive Gravity, Rev. Mod. Phys. 84 (2012) 671 [arXiv:1105.3735] [INSPIRE].

[38] C. de Rham, Galileons in the Sky, Comptes Rendus Physique 13 (2012) 666 [arXiv: 1204.5492] [INSPIRE].

[39] C. de Rham and A.J. Tolley, DBI and the Galileon reunited, JCAP 05 (2010) 015 [arXiv: 1003.5917] [INSPIRE].

[40] K. Hinterbichler, M. Trodden and D. Wesley, Multi-field galileons and higher co-dimension branes, Phys. Rev. D 82 (2010) 124018 [arXiv: 1008.1305] [INSPIRE].

[41] G. Goon, K. Hinterbichler and M. Trodden, Symmetries for Galileons and DBI scalars on curved space, JCAP 07 (2011) 017 [arXiv: 1103.5745] [INSPIRE].

[42] G. Goon, K. Hinterbichler and M. Trodden, A New Class of Effective Field Theories from Embedded Branes, Phys. Rev. Lett. 106 (2011) 231102 [arXiv:1103.6029] [INSPIRE].

[43] A.I. Vainshtein, To the problem of nonvanishing gravitation mass, Phys. Lett. B 39 (1972) 393 [INSPIRE].

[44] E. Babichev and C. Deffayet, An introduction to the Vainshtein mechanism, Class. Quant. Grav. 30 (2013) 184001 [arXiv: 1304.7240] [INSPIRE].

[45] G. Goon, K. Hinterbichler, A. Joyce and M. Trodden, Aspects of Galileon Non-Renormalization, JHEP 11 (2016) 100 [arXiv:1606.02295] [InSPIRE].

[46] G. Goon, K. Hinterbichler and M. Trodden, Galileons on Cosmological Backgrounds, JCAP 12 (2011) 004 [arXiv: 1109.3450] [INSPIRE].

[47] M. Trodden and K. Hinterbichler, Generalizing Galileons, Class. Quant. Grav. 28 (2011) 204003 [arXiv: 1104.2088] [INSPIRE].

[48] G. Goon, K. Hinterbichler, A. Joyce and M. Trodden, Galileons as Wess-Zumino Terms, JHEP 06 (2012) 004 [arXiv:1203.3191] [InSPIRE].

[49] G. Dvali, A. Gruzinov and M. Zaldarriaga, The accelerated universe and the moon, Phys. Rev. D 68 (2003) 024012 [hep-ph/0212069] [INSPIRE].

[50] A. Adams, N. Arkani-Hamed, S. Dubovsky, A. Nicolis and R. Rattazzi, Causality, analyticity and an IR obstruction to UV completion, JHEP 10 (2006) 014 [hep-th/0602178] [INSPIRE]. 
[51] G.L. Goon, K. Hinterbichler and M. Trodden, Stability and superluminality of spherical DBI galileon solutions, Phys. Rev. D 83 (2011) 085015 [arXiv: 1008.4580] [INSPIRE].

[52] M. Andrews, K. Hinterbichler, J. Khoury and M. Trodden, Instabilities of Spherical Solutions with Multiple Galileons and $\mathrm{SO}(N)$ Symmetry, Phys. Rev. D 83 (2011) 044042 [arXiv: 1008.4128] [INSPIRE].

[53] J. Evslin and T. Qiu, Closed Timelike Curves in the Galileon Model, JHEP 11 (2011) 032 [arXiv:1106.0570] [INSPIRE].

[54] T.L. Curtright and D.B. Fairlie, A Galileon Primer, arXiv:1212.6972 [INSPIRE].

[55] P. de Fromont, C. de Rham, L. Heisenberg and A. Matas, Superluminality in the Bi- and Multi-Galileon, JHEP 07 (2013) 067 [arXiv:1303.0274] [INSPIRE].

[56] S. Garcia-Saenz, Behavior of perturbations on spherically symmetric backgrounds in multi-Galileon theory, Phys. Rev. D 87 (2013) 104012 [arXiv: 1303.2905] [INSPIRE].

[57] L. Berezhiani, G. Chkareuli and G. Gabadadze, Restricted Galileons, Phys. Rev. D 88 (2013) 124020 [arXiv: 1302.0549] [INSPIRE].

[58] G. Gabadadze, R. Kimura and D. Pirtskhalava, Vainshtein Solutions Without Superluminal Modes, Phys. Rev. D 91 (2015) 124024 [arXiv:1412.8751] [INSPIRE].

[59] K. Hinterbichler, A. Nicolis and M. Porrati, Superluminality in DGP, JHEP 09 (2009) 089 [arXiv:0905.2359] [INSPIRE].

[60] S. Deser, A. Waldron and G. Zahariade, Propagation peculiarities of mean field massive gravity, Phys. Lett. B 749 (2015) 144 [arXiv:1504.02919] [INSPIRE].

[61] P. Creminelli, M. Serone, G. Trevisan and E. Trincherini, Inequivalence of Coset Constructions for Spacetime Symmetries, JHEP 02 (2015) 037 [arXiv:1403.3095] [inSPIRE].

[62] S.D. Mathur, The information paradox: A pedagogical introduction, Class. Quant. Grav. 26 (2009) 224001 [arXiv:0909.1038] [INSPIRE].

[63] S.D. Mathur, The fuzzball proposal for black holes: An elementary review, Fortsch. Phys. 53 (2005) 793 [hep-th/0502050] [INSPIRE].

[64] G. Dvali and C. Gomez, Black Hole's Quantum N-Portrait, Fortsch. Phys. 61 (2013) 742 [arXiv:1112.3359] [INSPIRE].

[65] A. Almheiri, D. Marolf, J. Polchinski and J. Sully, Black Holes: Complementarity or Firewalls?, JHEP 02 (2013) 062 [arXiv:1207.3123] [INSPIRE].

[66] G. Dvali, G.F. Giudice, C. Gomez and A. Kehagias, UV-Completion by Classicalization, JHEP 08 (2011) 108 [arXiv:1010.1415] [InSPIRE].

[67] L. Keltner and A.J. Tolley, UV properties of Galileons: Spectral Densities, arXiv: 1502.05706 [INSPIRE].

[68] R. Klein, M. Ozkan and D. Roest, Galileons as the Scalar Analogue of General Relativity, Phys. Rev. D 93 (2016) 044053 [arXiv:1510.08864] [inSPIRE].

[69] S.M. Carroll, Spacetime and geometry: An introduction to general relativity, Addison Wesley (2004).

[70] C.W. Misner, K.S. Thorne and J.A. Wheeler, Gravitation, W.H. Freeman, San Francisco, U.S.A. (1973). 
[71] N. Arkani-Hamed, L. Motl, A. Nicolis and C. Vafa, The string landscape, black holes and gravity as the weakest force, JHEP 06 (2007) 060 [hep-th/0601001] [INSPIRE].

[72] X.O. Camanho, J.D. Edelstein, J. Maldacena and A. Zhiboedov, Causality Constraints on Corrections to the Graviton Three-Point Coupling, JHEP 02 (2016) 020 [arXiv:1407.5597] [INSPIRE].

[73] K. Benakli, S. Chapman, L. Darmé and Y. Oz, Superluminal graviton propagation, Phys. Rev. D 94 (2016) 084026 [arXiv:1512.07245] [INSPIRE].

[74] J. Jing, S. Chen and Q. Pan, Geometric optics for a coupling model of electromagnetic and gravitational fields, Annals Phys. 367 (2016) 219 [arXiv:1510.03316] [INSPIRE].

[75] R. Penrose, On Schwarzschild Causality - A Problem for "Lorentz Covariant" General Relativity, in Essays in General Relativity: A Festschrift for Abraham Taub, Academic Press (1980).

[76] S. Gao and R.M. Wald, Theorems on gravitational time delay and related issues, Class. Quant. Grav. 17 (2000) 4999 [gr-qc/0007021] [INSPIRE].

[77] S.D. Majumdar, A class of exact solutions of Einstein's field equations, Phys. Rev. 72 (1947) 390 [INSPIRE].

[78] A. Papaetrou, A static solution of the equations of the gravitational field for an arbitrary charge distribution, Proc. Roy. Irish Acad. A 51 (1947) 191 [InSPIRE].

[79] J.B. Hartle and S.W. Hawking, Solutions of the Einstein-Maxwell equations with many black holes, Commun. Math. Phys. 26 (1972) 87 [INSPIRE].

[80] M.D. Schwartz, Quantum Field Theory and the Standard Model, Cambridge University Press, (2014).

[81] E.T. Akhmedov, H. Godazgar and F.K. Popov, Hawking radiation and secularly growing loop corrections, Phys. Rev. D 93 (2016) 024029 [arXiv:1508.07500] [InSPIRE].

[82] A. Kamenev, Field Theory of Non-Equilibrium Systems, Cambridge University Press, (2011).

[83] S. Weinberg, Quantum contributions to cosmological correlations, Phys. Rev. D 72 (2005) 043514 [hep-th/0506236] [INSPIRE].

[84] M.J. Duff, Quantum Tree Graphs and the Schwarzschild Solution, Phys. Rev. D 7 (1973) 2317 [INSPIRE].

[85] M.J. Duff, Quantum corrections to the Schwarzschild solution, Phys. Rev. D 9 (1974) 1837 [INSPIRE].

[86] G.W. Gibbons, Vacuum Polarization and the Spontaneous Loss of Charge by Black Holes, Commun. Math. Phys. 44 (1975) 245 [InSPIRE].

[87] G. Goon and K. Hinterbichler, in preparation.

[88] G. 't Hooft and M.J.G. Veltman, One loop divergencies in the theory of gravitation, Annales Poincare Phys. Theor. A 20 (1974) 69.

[89] M.H. Goroff and A. Sagnotti, The Ultraviolet Behavior of Einstein Gravity, Nucl. Phys. B 266 (1986) 709 [INSPIRE].

[90] D.M. Capper, M.J. Duff and L. Halpern, Photon corrections to the graviton propagator, Phys. Rev. D 10 (1974) 461 [INSPIRE]. 
[91] J.F. Donoghue, B.R. Holstein, B. Garbrecht and T. Konstandin, Quantum corrections to the Reissner-Nordstrom and Kerr-Newman metrics, Phys. Lett. B 529 (2002) 132 [Erratum ibid. B 612 (2005) 311] [hep-th/0112237] [INSPIRE].

[92] N.E.J. Bjerrum-Bohr, J.F. Donoghue and B.R. Holstein, Quantum corrections to the Schwarzschild and Kerr metrics, Phys. Rev. D 68 (2003) 084005 [Erratum ibid. D 71 (2005) 069904] [hep-th/0211071] [INSPIRE].

[93] G.G. Kirilin, Quantum corrections to the Schwarzschild metric and reparametrization transformations, Phys. Rev. D 75 (2007) 108501 [gr-qc/0601020] [INSPIRE].

[94] C.P. Burgess, Quantum gravity in everyday life: General relativity as an effective field theory, Living Rev. Rel. 7 (2004) 5 [gr-qc/0311082] [INSPIRE].

[95] J.F. Donoghue, General relativity as an effective field theory: The leading quantum corrections, Phys. Rev. D 50 (1994) 3874 [gr-qc/9405057] [INSPIRE].

[96] L.F. Abbott, Introduction to the Background Field Method, Acta Phys. Polon. B 13 (1982) 33 [INSPIRE].

[97] D.A.R. Dalvit and F.D. Mazzitelli, Geodesics, gravitons and the gauge fixing problem, Phys. Rev. D 56 (1997) 7779 [hep-th/9708102] [INSPIRE].

[98] A.O. Barvinsky and G.A. Vilkovisky, The Generalized Schwinger-Dewitt Technique in Gauge Theories and Quantum Gravity, Phys. Rept. 119 (1985) 1 [InSPIRE].

[99] A. Andreassen, W. Frost and M.D. Schwartz, Consistent Use of Effective Potentials, Phys. Rev. D 91 (2015) 016009 [arXiv: 1408.0287] [InSPIRE].

[100] N.K. Nielsen, On the Gauge Dependence of Spontaneous Symmetry Breaking in Gauge Theories, Nucl. Phys. B 101 (1975) 173 [INSPIRE].

[101] R. Fukuda and T. Kugo, Gauge Invariance in the Effective Action and Potential, Phys. Rev. D 13 (1976) 3469 [INSPIRE].

[102] I.J.R. Aitchison and C.M. Fraser, Gauge Invariance and the Effective Potential, Annals Phys. 156 (1984) 1 [INSPIRE].

[103] N.E.J. Bjerrum-Bohr, J.F. Donoghue and B.R. Holstein, Quantum gravitational corrections to the nonrelativistic scattering potential of two masses, Phys. Rev. D 67 (2003) 084033 [Erratum ibid. D 71 (2005) 069903] [hep-th/0211072] [INSPIRE].

[104] I.B. Khriplovich and G.G. Kirilin, Quantum power correction to the Newton law, J. Exp. Theor. Phys. 95 (2002) 981 [gr-qc/0207118] [INSPIRE].

[105] B.R. Holstein and A. Ross, Long Distance Effects in Mixed Electromagnetic-Gravitational Scattering, arXiv:0802.0717 [INSPIRE].

[106] N.E.J. Bjerrum-Bohr, Leading quantum gravitational corrections to scalar QED, Phys. Rev. D 66 (2002) 084023 [hep-th/0206236] [INSPIRE].

[107] S.L. Adler, Photon splitting and photon dispersion in a strong magnetic field, Annals Phys. 67 (1971) 599 [INSPIRE].

[108] A.K. Harding and D. Lai, Physics of Strongly Magnetized Neutron Stars, Rept. Prog. Phys. 69 (2006) 2631 [astro-ph/0606674] [INSPIRE].

[109] R. Ruffini, Y.-B. Wu and S.-S. Xue, Einstein-Euler-Heisenberg Theory and charged black holes, Phys. Rev. D 88 (2013) 085004 [arXiv:1307.4951] [InSPIRE]. 
[110] H. Yajima and T. Tamaki, Black hole solutions in Euler-Heisenberg theory, Phys. Rev. D 63 (2001) 064007 [gr-qc/0005016] [INSPIRE].

[111] Y. Kats, L. Motl and M. Padi, Higher-order corrections to mass-charge relation of extremal black holes, JHEP 12 (2007) 068 [hep-th/0606100] [INSPIRE].

[112] D. Marolf and J. Polchinski, Gauge/Gravity Duality and the Black Hole Interior, Phys. Rev. Lett. 111 (2013) 171301 [arXiv:1307.4706] [INSPIRE].

[113] W. Heisenberg and H. Euler, Consequences of Dirac's theory of positrons, Z. Phys. 98 (1936) 714 [physics/0605038] [INSPIRE]. 